\title{
Dislocation-actuated Growth and Inhibition of Hexagonal L-cystine Crystallization at the Molecular Level
}

Alexander G. Shtukenberg,,"* Laura N. Poloni," Zina Zhu, ${ }^{\#}$ Zhihua An, ${ }^{\#}$ Misha Bhandari,, Pengcheng Song, ${ }^{\#}$ Andrew L. Rohl, ${ }^{\$}$ Bart Kahr," ${ }^{\#}$ and Michael D. Ward ${ }^{\#, *}$

"Molecular Design Institute and Department of Chemistry, 100 Washington Square East, Silver Center, Room 1001, New York University, New York, NY 10003, USA

${ }^{\$}$ Nanochemistry Research Institute and Department of Chemistry, Curtin University, PO Box U1987, Perth, WA 6845, Australia

\begin{abstract}
Crystallization of L-cystine is a critical process in the pathogenesis of kidney stone formation in cystinuria, a disorder affecting more than 20,000 individuals in the U.S. alone. In an effort to elucidate the crystallization of $L$-cystine and the mode of action of tailored growth inhibitors that may constitute effective therapies, real-time, in situ atomic force microscopy (AFM) has been used to investigate the surface micromorphology and growth kinetics of the $\{0001\}$ faces of $L$-cystine at various supersaturations and concentrations of the growth inhibitor L-cystine dimethylester (CDME). Crystal growth is actuated by screw dislocations on the $\{0001\}$ L-cystine surface, producing hexagonal spiral hillocks that are a consequence of six interlacing spirals of anisotropic molecular layers. The high level of elastic stress in the immediate vicinity around the dislocation line results in a decrease in the step velocities and a corresponding increase in the spacing of steps. The kinetic curves acquired in the presence of CDME conform to the classical Cabrera-Vermilyea model. Anomalous birefringence in the $\{1010\}$ growth sectors, combined with computational modeling, supports a high fidelity of stereospecific binding of $\mathrm{CDME}$, in a unique orientation, exclusively at one of the six crystallographically unique projections on the $\{10 \overline{10}\}$ plane.
\end{abstract}

Keywords: L-cystine, atomic force microscopy, cystinuria, crystal growth, dislocation

*To whom correspondence should be addressed. Email: shtukenberg@mail.ru; mdw3@nyu.edu 


\section{INTRODUCTION}

Cystinuria, a genetic disorder that afflicts more than 20,000 individuals in the U.S. alone, is associated with abnormal levels of $L$-cystine in the kidney and is often accompanied by recurring formation of $L$ cystine stones. Current treatments for $L$-cystine stone prevention include dilution through high fluid intake, ${ }^{1}$ increasing urine $\mathrm{pH}$ through ingestion of alkalinizing potassium or sodium salts, ${ }^{1,2}$ or the administration of $L$-cystine binding thiol drugs (CBTDs). ${ }^{3}$ These treatments suppress, but typically do not completely prevent, stone formation. Moreover, CBTDs do not reduce $L$-cystine concentrations sufficiently at dosages regarded as below the threshold for hypersensitivity and toxicity. Our laboratory recently demonstrated that low concentrations of certain additives - methyl esters of $L$-cystine that mimic the structure of $L$-cystine - inhibited the formation of $L$-cystine crystals, suggesting a new approach to a therapy for this disease. ${ }^{4}$ Real-time in situ atomic force microscopy (AFM) of $L$-cystine crystals in supersaturated $L$-cystine solutions revealed growth hillocks emanating from screw dislocations on the (0001) face. The micromorphology of these hillocks was attributed to interlaced spirals of anisotropic molecular layers of $L$-cystine bunching in a manner that deceptively appeared as stacks of hexagonal islands. The addition of the $L$-cystine methyl esters to the growth solution resulted in roughening of the hillock steps as well as a decrease in step velocity. These observations suggested that step pinning by "molecular imposters," sometimes referred to as tailor-made auxiliaries that are known to affect crystal morphology, ${ }^{5-7}$ was responsible for growth inhibition. Further investigation of the molecular mechanisms responsible for these phenomena may lead to advances in the design of new therapeutic agents for the prevention of $L$-cystine stones. Moreover, the ease of locating dislocations on the $L$-cystine (0001) surface and the ability to use AFM for quantitative measurements of step velocities provides a unique opportunity to test crystal growth theories and inhibition at the near-molecular level, expanding on related

investigations of crystal growth that have largely been limited to inorganic and macromolecular crystals. ${ }^{8-}$ 10

Herein, we describe AFM investigations of the micromorphology of dislocation spirals on the (0001) face of hexagonal $L$-cystine crystals, quantitative measurements of kinetic coefficients, critical length, and step spacing, and the influence of the inhibitor L-cystine dimethylester (CDME) on these features. The high level of elastic stress in the vicinity of the dislocation outcrop increases the effective solubility, as surmised from step spacings larger than those expected from classical spiral growth models. Growth inhibition by CDME is consistent with a Cabrera-Vermilyea step-pinning mechanism, and anomalous birefringence in the $\{10 \overline{10}\}$ growth sectors, combined with computational modeling, supports a high 
fidelity of stereospecific binding of CDME, in a unique orientation, exclusively at one of the six crystallographically unique projections on the $\{10 \overline{10}\}$ plane.

\section{EXPERIMENTAL SECTION}

Preparation of hexagonal $\boldsymbol{L}$-cystine crystals. All crystallization experiments were performed at $\mathrm{pH}=$ 6.3. Under these conditions $L$-cystine crystallizes as hexagonal platelets expressing $\{0001\}$ and $\{10 \overline{0}\}$ faces. ${ }^{11}$ The hexagonal modification, in contrast to the tetragonal polymorph, was crystallized from a supersaturated $L$-cystine solution prepared by adding $70 \mathrm{mg}$ of $L$-cystine to $100 \mathrm{~mL}$ of deionized water (3 $\mathrm{mM}$ ), followed by heating under reflux at $100{ }^{\circ} \mathrm{C}$ for $30 \mathrm{~min}$ with stirring so that the $L$-cystine dissolved completely. The resulting solution corresponds to a relative supersaturation $C / C_{e q} \approx 5$, based on the reported solubility $\left(C_{e q}=0.7 \mathrm{mM}\right.$ at $\left.\mathrm{pH} 7,25^{\circ} \mathrm{C}^{; 12-14}\right)$. The value $C_{e q}=0.7 \mathrm{mM}$ was confirmed by our AFM measurements (Figure 7B). The solution was then allowed to cool slowly with stirring for 75 min, after which $30 \mathrm{~mL}$ aliquots were transferred to separate glass containers, which were then sealed (to prevent evaporation and exposure to airborne particulates) and stored for 72 hours at room temperature without stirring. Single crystals were collected by vacuum filtration (Whatman Grade 1 filters, $>11 \mu \mathrm{m}$ pores) and air-dried prior to AFM experiments. Bulk crystallization of $L$-cystine in the presence of CDME was performed by adding the required amount of CDME to a $200 \mathrm{~mL}$ aliquot of the supersaturated $L$ cystine solution that had been allowed to cool for 75 minutes, prior to observable crystallization.

In situ AFM measurements of crystal growth. Hexagonal $L$-cystine crystals, prepared by the procedure above, were transferred onto an AFM specimen disk coated with partially cured (1 min) Norland optical adhesive (Type 81 ) by gently pressing the disk against hexagonal platelets collected by filtration (Whatman Nuclepore membrane, $8 \mu \mathrm{m}$ ). The (0001) faces of the hexagonal plates naturally aligned parallel to the specimen disk, which allowed facile measurement of $\{10 \overline{10}\}$ step velocities. The adhered crystals were affixed permanently by curing the adhesive completely with additional UV radiation (15 $\min )$. The mounted $L$-cystine crystals were etched slightly by immersion in deionized water for $30 \mathrm{sec}$ at $65{ }^{\circ} \mathrm{C}$ to remove any amorphous deposits or impurities present on the surface. In situ AFM was performed at room temperature with a Digital Instruments (Santa Barbara, CA) Nanoscope IIIa Multimode system to collect sequential images of growing crystals. All measurements were performed in a $0.5 \mathrm{~mL}$ cell designed to contain liquids, using contact mode and Veeco NP-B $\mathrm{Si}_{3} \mathrm{~N}_{4}$ tips on silicon nitride cantilevers with a spring constant of $0.12 \mathrm{~N} / \mathrm{m}$ (triangular, $196 \mu \mathrm{m}$ length, $41 \mu \mathrm{m}$ width). 
Prior to measuring step velocities in the presence of CDME, the AFM fluid cell was flushed with a $2 \mathrm{mM}$ $L$-cystine solution to regenerate the crystal surface and to confirm the step velocity at this concentration. This and all subsequent measurements were performed under a continuous flow of $L$-cystine solution (injected into the AFM cell with a syringe pump at a rate of $10 \mathrm{~mL} / \mathrm{h}$ ) in order to maintain constant supersaturation and additive concentration. The velocities of step bunches, $V_{\mathrm{A}^{*}}$, were determined by measuring the distance of the steps from a reference point, usually the dislocation core, in consecutive deflection images acquired at periodic intervals that ranged from 9 - $14 \mathrm{sec}$. The steps advanced linearly with time. The standard deviation was calculated from the average of the step velocities determined for three different steps on the same terrace of a given crystal. Velocities of the minor $(c / 6)$ steps, corresponding to a single molecular layer, in the core region $\left(V_{\mathrm{A}(+)}, V_{\mathrm{B}(+)}, . . V_{\mathrm{C}(-)}\right)$ were determined according to the procedure illustrated in Figure 2C,D and described in its caption.

Step edge root-mean-square (RMS) roughness. The step edge RMS roughness was calculated from AFM images, acquired in height mode, as $R=\sqrt{\left(\sum_{i=1}^{N}\left(y_{i}-y_{\mathrm{av}}\right)^{2}\right) / N}$, where $y_{i}$ is the displacement of the step edge from the average position $y_{\mathrm{av}}$, evaluated from the linear fit for $N$ points measured for a given edge. The RMS roughness was determined from the average of measurements on six differently oriented steps located outside the dislocation core ( $1-2 \mu \mathrm{m}$ from the center of a common dislocation hillock).

Optical microscopy. Anomalous birefringence viewed along [0001] direction of $L$-cystine crystals was imaged with an Olympus BX50 polarized light microscope equipped with a digital camera. The linear retardance and the extinction directions were determined by the rotating polarizer technique, as implemented in a prototype of the Metripol microscope. ${ }^{15,16}$

Nuclear magnetic resonance (NMR). The total CDME concentration included in $L$-cystine crystals through overgrowth was measured by ${ }^{1} \mathrm{H}$ NMR of solutions consisting of crystals dissolved in $1 \mathrm{M} \mathrm{DCl}$. Spectra were acquired with a Bruker AVANCE 400 spectrometer and the residual solvent proton resonance of $\mathrm{D}_{2} \mathrm{O}$ at $4.79 \mathrm{ppm}$ was taken as the reference.

Computations. Geometry optimizations and single-point energy calculations were conducted using the Forcite molecular mechanics tool within Accelrys Materials Studio ${ }^{\circledR}$ v 4.2. The Quasi-Newton algorithm was used for all geometry optimizations with a convergence tolerance of $2.0 \times 10^{-5} \mathrm{kcal} / \mathrm{mol}$ for the energy, $0.001 \mathrm{kcal} / \mathrm{mol} / \AA$ for the force and $1.0 \times 10^{-5} \AA$ for the displacement. The COMPASS forcefield ${ }^{17}$ was used in all calculations with forcefield-assigned partial charges. The Ewald summation method was 
chosen for the evaluation of van der Waals and electrostatic terms to an accuracy of $0.0001 \mathrm{kcal} / \mathrm{mol}$ with a buffer width of $0.5 \AA$.

Stepped $L$-cystine surfaces were created by importing the hexagonal $L$-cystine crystallographic information file into Materials Studio, inserting bonds between the atoms, and optimizing the unit cell. (0001) $L$-cystine surfaces were created by cleaving the $L$-cystine unit cell to a fractional depth of 0.67 (four $L$-cystine molecules) and constructing an $8 \times 8(43.36 \AA \times 43.36 \AA$ ) supercell. Rows of surface molecules were manually deleted to create steps in all six $\{1010\}$ orientations. A vacuum slab of $100 \AA$ was inserted above each stepped surface structure and 3D boundary conditions were applied. Solvation energies were calculated using the General Utility Lattice Program (GULP), version 4.0.3. ${ }^{18}$ The various $L$-cystine structures (stepped surfaces, isolated rows) were solvated using the COSMIC model ${ }^{19}$ with a dielectric constant of 78.4 and a solvent radius of $1.4 \AA$, a delta solvent radius of $1.3 \AA$, and a cutoff of 10 $\AA$, with 194 points per sphere.

Binding energies associated with the attachment of an infinite row of $L$-cystine molecules to infinite steps along each of the six $\{10 \overline{10}\}$ growth directions $\left(E_{\mathrm{b}}\right)$ (Table 1) were calculated from single-point energy calculations and solvation energies. Single-point energy calculations were performed on stepped surfaces with steps oriented along one of the six $\{1010\}$ growth orientations $\left(E_{\text {step }}\right)$. Stepped surfaces in each orientation were built in a similar manner with an additional row of $L$-cystine molecules at the step site, and single-point energy calculations were again performed $\left(E_{\text {step+row }}\right)$. The additional rows of molecules were isolated and single-point energy calculations were performed $\left(E_{\text {row }}\right)$. Solvation energies for the supercell structures associated with $E_{\text {step }}, E_{\text {row }}$, and $E_{\text {step+row }}$ for each orientation were calculated subsequently. Binding energies of infinite rows to steps along the six growth orientations of $L$-cystine were calculated by

$$
E_{\mathrm{b}}=\left(\left[E_{\text {step }+ \text { row }}+E_{\text {step }+ \text { row, solv }}\right]-\left[\left(E_{\text {step }}+E_{\text {step, solv }}\right)+\left(E_{\text {row }}+E_{\text {row }, \text { solv }}\right)\right]\right) / N .
$$

where $N$ is the number of molecules in the row binding to the step site per supercell ( $N=8$ in this case).

CDME incorporation into a $L$-cystine crystal was modeled by replacement of a $L$-cystine molecule in the crystal structure. A $5 a \times 4 b \times 1 c$ supercell was created from the $L$-cystine unit cell and an incorporated CDME molecule was created by replacing the terminal carboxylate groups of a single $L$-cystine molecule with methyl esters. The amine groups on the $L$-cystine backbone of the CDME molecule were deprotonated to maintain charge neutrality and 3D boundary conditions were applied to model an infinite crystal. The geometry was optimized with all molecules surrounding the CDME impurity molecule 
allowed to relax. The unconstrained portion of supercell measured $3 a \times 4 b \times 1 / 2 c$ (36 of the 120 molecules).

Binding energies of CDME to the six crystallographically unique orientations of $L$-cystine layers along the $\{1010\}$ growth face were calculated as replacement energies $\left(E_{\text {repl }}\right)$, with CDME binding stereospecifically at each crystal binding site. The $\{10 \overline{10}\}$ growth surface was constructed by cleaving the hexagonal unit cell parallel to the (1010) growth plane to a depth of six unit cells (26.84 $\AA$ ). A $1 v$ x $4 u$ (56.28 $\AA 21.68 \AA$ ) supercell was constructed and a $100 \AA$ vacuum slab was constructed above the (1010) surface. The conformation of the single unconstrained CDME or $L$-cystine molecule at each binding site was optimized with all surrounding $L$-cystine molecules constrained. Single-point energy calculations were then performed on each of the simulation cells to determine the total energy of the CDME- and $L$ cystine-incorporated systems $\left(E_{\mathrm{CDME} \text {-incorporated surface }}\right.$ and $\left.E_{L \text {-cystine surface }}\right)$. Isolated $L$-cystine and $\mathrm{CDME}$ molecules were optimized and their energies calculated ( $E_{L \text {-cystine }}$ and $\left.E_{\mathrm{CDME}}\right)$. Replacement energies $\left(E_{\text {repl }}\right)$ were calculated as

$$
E_{\text {repl }}=\left(E_{\mathrm{CDME} \text {-incorporated surface }}+E_{L \text {-cystine }}\right)-\left(E_{L \text {-cystine surface }}+E_{\mathrm{CDME}}\right) \text {. }
$$

Elastic constants were independently calculated using two different programs distinguished by different force fields; GULP was used with the GAFF forcefield ${ }^{20}$ with RESP-A1A charges ${ }^{21}$ and Materials Studio with the COMPASS forcefield. GULP calculates the elastic constants analytically, whereas Materials Studio employs a numerical algorithm.

\section{RESULTS AND DISCUSSION}

Dislocation spirals on $\{0001\} \boldsymbol{L}$-cystine faces. Real time, in situ atomic force microscopy (AFM) was used to investigate the crystal growth modes on the (0001) hexagonal $L$-cystine (noncentrosymmetric space group $\left.P 6_{1} 22\right)^{22}$ surface in solutions supersaturated with $L$-cystine $\left(L\right.$-cystine solubility $C_{e q}=0.7$ $\mathrm{mM}$ ). Spontaneous nucleation on the crystal surface was observed consistently at concentrations greater than $3.5 \mathrm{mM}$, precluding AFM measurements of spiral growth. In the concentration range $0.7 \mathrm{mM}<C<$ $3.5 \mathrm{mM}$, however, spiral growth on the $\{0001\} L$-cystine face dominates, as reported previously by our laboratory. ${ }^{4}$ Under these conditions the $\{0001\}$ surface was characterized by hexagonal growth hillocks that resemble stacks of islands. Each island was approximately $5.6 \mathrm{~nm}$ high, corresponding to the $c$ unit cell length $(c=5.6275 \mathrm{~nm})$. The $6_{1}$ screw axis inherent to the space group symmetry is expressed as a pinwheel of minor steps, successively rotated clockwise around the $c$ axis by $60^{\circ}$ that spin out from each island below. The height of these minor steps is $c a$. $1 \mathrm{~nm}$, equivalent to $c / 6$, the thickness of one of six elementary layers in the crystal structure that stack along the $c$ axis and equivalent to the height of a 
cystine molecule (Figure 1A). The magnitude of the Burgers vector normal to the $\{0001\}$ face is $c=$ $5.6275 \mathrm{~nm},{ }^{22}$ and the observation of the $c / 6$ steps is tantamount to step splitting. ${ }^{23,24}$ The handedness of the spiral hillocks on the $\{0001\}$ faces of $L$ - and $D$-cystine reflects the chirality at the molecular level; the spirals spin counterclockwise on $L$-cystine $\{0001\}$ but clockwise on $D$-cystine $\{0001\}$ (based on examination of $80 \mathrm{~L}$-cystine spirals and $5 \mathrm{D}$-cystine spirals). A similar preference for the handedness of spirals has been observed for other enantiomeric crystals, including amino acids. ${ }^{25}$ Although the results described herein are based on data acquired for $L$-cystine, the behavior of $D$-cystine was found to be identical in every respect except spiral handedness. We note, however, that pairs of screw dislocations of opposite chiralities (i.e. Frank-Read sources) also have been observed on the $\{0001\}$ face of $L$-cystine. Although the origin of spiral handedness is not fully understood, it is reasonable to suggest that the handedness reflects discrimination during the initial stages of dislocation generation. 


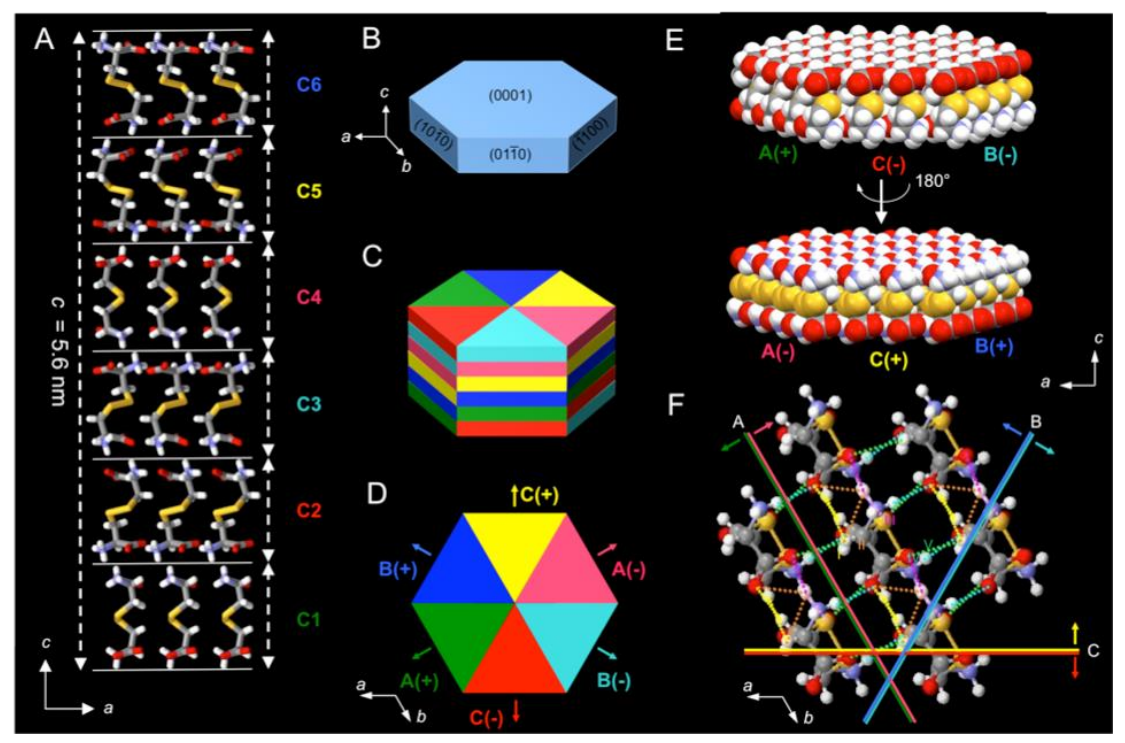

Figure 1. (A) Three adjacent helices of $L$-cystine molecules, as viewed along the [0110] direction (i.e. the $b$ axis), each winding about a 6 screw axis along the [0001] direction (i.e. the $c$ axis). Six layers of $L$-cystine molecules, denoted $\mathrm{C} 1$ to $\mathrm{C} 6$, span the $5.6 \mathrm{~nm} C$ axis, resulting in six unique presentations of $L$-cystine molecules on each of the six growth faces. (B) Schematic illustration of a hexagonal L-cystine crystal with Miller indices. (C) Schematic illustration of a hexagonal crystal with a height spanning six molecular layers. The colors correspond to different orientations of $L$-cystine at each face. For example, green denotes a unique orientation of $L$-cystine on the (1010) face in layer $\mathrm{C} 1$, on the (0110) face in layer $\mathrm{C} 2$, etc., winding around the $c$ axis according to the $6_{1}$ screw axis symmetry. (D) Schematic illustration of the $C 1$ layer viewed along the $c$ axis. The six different presentations of $L$ cystine, denoted $\mathrm{A}(+), \mathrm{B}(+), \mathrm{C}(+), \mathrm{A}(-), \mathrm{B}(-)$, and $\mathrm{C}(-)$, correspond to the edges of a $c / 6$ molecular layer and their growth directions (as observed by AFM, see below). (E) Space-filling model of a single cystine $c / 6$ layer. Atom colors: carbon (gray), oxygen (red), nitrogen (blue), sulfur (yellow), hydrogen (white). (F) The crystal structure of a C1 layer as viewed along the $c$ axis. $\mathrm{A}, \mathrm{B}$, and $\mathrm{C}$ correspond to slices regarded as the most reasonable based on HartmanPerdok theory. Intermolecular amine-carboxylate hydrogen bonds in the (0001) plane are highlighted in colored

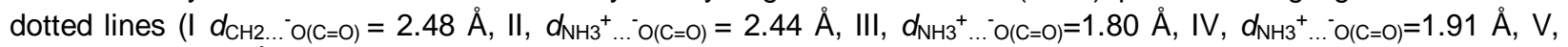
$d_{\mathrm{CH} 2 \ldots(\mathrm{C}=\mathrm{O})}=2.64 \AA$ ) . Single crystal structural data retrieved from the Cambridge Structural Database; REFCODE LCYSTI10, Reference 22.

The $c / 6$ molecular layers of $L$-cystine contain a two-fold axis in-plane, but lack symmetry elements (e.g., rotation, mirror) parallel to [0001], resulting in a structural anisotropy. Each layer, denoted C1 through C6 in Figure 1A, is bounded by six inequivalent edges aligned with the $\langle 10 \overline{10}\rangle$ directions, defining six minor step planes with unique surface energies that anticipate different step lengths and growth velocities. According to Hartman-Perdok theory, ${ }^{26-28}$ steps that sever the fewest strong bonds have the lowest surface energy. The step velocity is expected to scale with the step surface energy, that is, the steps with the lowest surface energy are expected to exhibit the slowest step velocities. Using the molecular layer arbitrarily assigned $\mathrm{C} 1$ as illustrative, analysis of the intermolecular hydrogen bonds suggests that the 
lowest energy steps are parallel to $\{1010\}$ planes, corresponding to slices A, B, and C in Figure $1 \mathrm{~F}$. These minor steps can advance in either the forward or reverse direction, leading to six unique growth directions, denoted $\mathrm{A}(+), \mathrm{A}(-), \mathrm{B}(+), \mathrm{B}(-), \mathrm{C}(+)$, and $\mathrm{C}(-)$ (Figure 1D). Minor step A contains two in-plane N-H...O hydrogen bond pairs $(1.80 \AA)$ and short S...S contacts $(3.47 \AA)$ along the [1120] direction and truncates the fewest hydrogen bonds (two N-H...O hydrogen bonds pairs (1.91 $\AA$ )). Minor steps B and C, parallel to other $\langle 11 \overline{2} 0\rangle$ directions, each truncate four N-H...O hydrogen bond pairs, (two stronger bond pairs, $1.80 \AA$, and two weaker bond pairs, $1.91 \AA$ ), suggesting a higher step surface energy and velocity of these steps compared with step A.

This qualitative step energy ranking based on hydrogen bond counting was corroborated by calculation of the binding energies of rows of $L$-cystine molecules to the six unique molecular steps, either in vacuum or with implicit solvation, the latter treating solvent as a continuous medium rather than as individual solvent molecules. Calculations could not discriminate between opposite growth directions within a pair $(\mathrm{A}(+) / \mathrm{A}(-), \mathrm{B}(+) / \mathrm{B}(-), \mathrm{C}(+) / \mathrm{C}(-))$, but the binding energies of minor steps $\mathrm{A}(+)$ and $\mathrm{A}(-)$ were smaller than the other four steps, consistent with a lower surface energy and suggesting lower step velocities (Table 1).

\begin{tabular}{|c|c|c|c|}
\hline \multirow[t]{2}{*}{$\begin{array}{l}\text { Miller } \\
\text { plane }\end{array}$} & \multirow[t]{2}{*}{$\begin{array}{c}\text { Step } \\
\text { orientation }\end{array}$} & \multicolumn{2}{|c|}{$\begin{array}{l}\text { Binding energy per } \\
\text { molecule (kcal/mol) }\end{array}$} \\
\hline & & Vacuum & Solvated \\
\hline (10̄10) & $A(+)$ & -43.8 & -22.4 \\
\hline$(\overline{\overline{1} 010})$ & $A(-)$ & -43.8 & -22.4 \\
\hline$(1 \overline{1} 00)$ & $B(+)$ & -98.3 & -36.2 \\
\hline$(\overline{1} 100)$ & $B(-)$ & -98.3 & -36.2 \\
\hline$(0 \overline{1} 10)$ & $C(+)$ & -98.3 & -35.9 \\
\hline$(01 \overline{1} 0)$ & $C(-)$ & -98.3 & -35.9 \\
\hline
\end{tabular}

As reported previously, ${ }^{29}$ the coincidence of the screw dislocation axis and crystallographic screw axis in hexagonal $L$-cystine results in six-threaded interlaced spirals, as observed using real-time in situ AFM. As the six minor steps are generated and advance from the dislocation core, the slowest step limits the propagation of faster-advancing steps in the layers above, resulting in bunching of the minor steps to form the 5.6-nm major steps flanking the hexagonal islands. Consequently, the growth anisotropy of a single molecular layer cannot be deduced from the bunched edges. Instead, the mode and rate of growth of the six step edges enclosing a molecular layer requires inspection near the dislocation core, where step bunches have not yet formed and each edge of a molecular layer can be discerned (Figure 2; the number denotes a single molecular $c / 6$ layer and the letters correspond to the six growth directions). These steps 
near the core are often rounded and show significant variations in velocity at constant growth conditions, particularly at low supersaturations (Figure 3) that is characteristic of the Cabrera-Vermilyea step pinning by an adventitious adsorbing impurity. ${ }^{8}$ Impurity-free growth rates were therefore estimated from the maximum step velocity along a given direction for several crystals (Figure. 2C,D). The step velocities along each growth direction at three supersaturations investigated exhibit strong anisotropy (Table 2). As expected from the structural and computational analyses above, the step velocities of $\mathrm{A}(+)$ and $\mathrm{A}(-)$ were significantly smaller than those of the other four steps. The step velocities for $\mathrm{B}(+), \mathrm{B}(-), \mathrm{C}(+)$, and $\mathrm{C}(-)$ at $C \geq 1.2 \mathrm{mM}$ usually reflected a lower limit because the acquisition time for a single image frame was slow compared with the evolution of the spiral arms. Measurements of step velocities for the slowest segments, $\mathrm{A}(+)$ and $\mathrm{A}(-)$, were reliable and reproducible at all supersaturations, however. Notably, velocities of $\mathrm{A}(+)$ in the core region (labeled as $V_{\mathrm{A}(+)}$ ) are smaller than velocities measured outside the core region (labeled by $V_{\mathrm{A}^{*}}$ ), which can be attributed to the high level of elastic stress near the dislocation line (see below). Far from the hillock center, the velocity of the slowest step, $\mathrm{A}(+)$, is nearly identical to that of the step bunches, consistent with the advance of $\mathrm{A}(+)$ limiting the rate of advance of the other five steps and the absence of diffusion control on the crystallization kinetics.
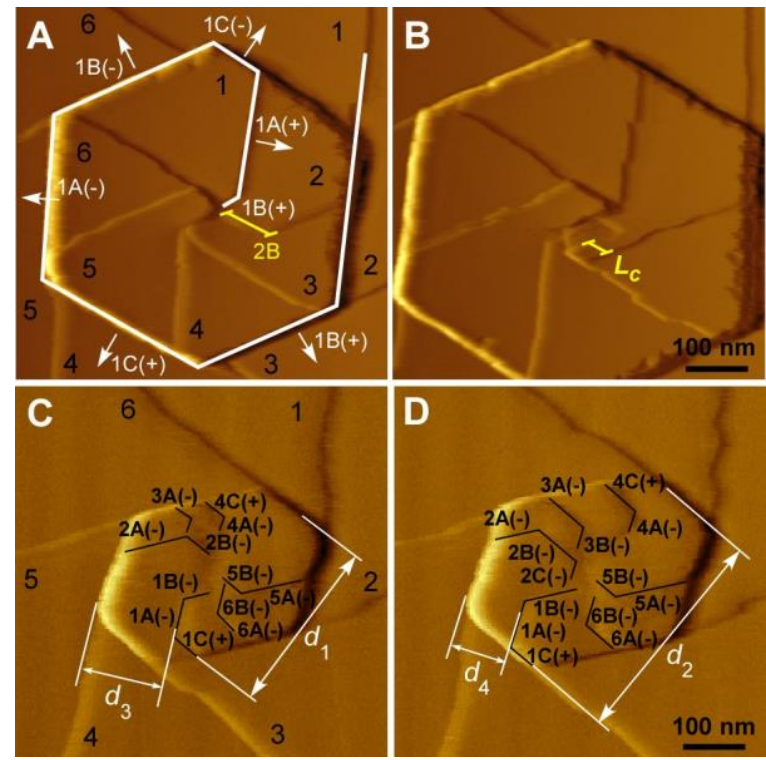

Figure 2. Growth hillocks on the L-cystine (0001) surface formed by a single dislocation. (A) Six unique growth steps are labeled from 1 to 6 and the six segment orientations for a single molecular layer are denoted by letters $A(+)$ to $C(-)$. Segment orientations for the edges of molecular layer 1 are highlighted by a white line. (B) The critical length, $L_{c}$, for $2 B(+)$. $L-c y s t i n e$ concentration $C=1.5 \mathrm{mM}$. (C, D) Step velocity measurements: $V_{1 \mathrm{C}(+)} \geq\left(d_{2}-d_{1}\right) / t-V_{\mathrm{A}^{*}} ; V_{1 \mathrm{~A}(-)}=\left(d_{3}-d_{4}\right) / t+V_{\mathrm{A}^{*}}$. L-cystine concentration $C=1.2 \mathrm{mM}$. The time intervals, $t$, between $(A)$ and $(B),(C)$ and $(D)$ are $5.25 \mathrm{~s}$. 
Table 2. Segment velocities, critical lengths, and some characteristics calculated for different $L$-cystine concentrations. $L_{c}$ values represent the minimum measured length for $\mathrm{B}(+)$ steps on several crystals. Segment orientations are shown in Figure $2 \mathrm{~A}$. Velocities of $A(+)$ inside the core region $(A(+))$ were smaller than velocities measured outside the core $\left(A^{*}\right)$.

\begin{tabular}{|c|c|c|c|c|c|c|c|c|c|c|c|c|}
\hline \multirow{2}{*}{$\begin{array}{c}C \\
\text { (mM) }\end{array}$} & \multirow{2}{*}{$\begin{array}{c}\Delta \mu_{0},= \\
(\mathrm{kJ} / \mathrm{mol})\end{array}$} & \multirow{2}{*}{$\begin{array}{c}\Delta \mu_{0}-\Delta \mu_{d} \\
(\mathrm{~kJ} / \mathrm{mol})\end{array}$} & \multicolumn{7}{|c|}{ Step velocity, $V(\mathrm{~nm} / \mathrm{s})$} & \multicolumn{2}{|c|}{$L_{c B}(\mathrm{~nm})$} & \multirow{2}{*}{$\begin{array}{l}r_{V=0} \\
(\mathrm{~nm})\end{array}$} \\
\hline & & & $A^{*}$ & $A(+)$ & $\mathrm{B}(+)$ & $C(+)$ & $A(-)$ & $B(-)$ & $C(-)$ & Meas. & Calc. & \\
\hline 1.2 & 1.31 & 0.45 & 2.3 & 1.8 & $\approx 13$ & 9.5 & 5.1 & 12.7 & 10.9 & 33 & 19 & 22 \\
\hline 1.5 & 1.85 & 1.00 & 4.4 & 2.1 & $>10.6$ & $>14.3$ & 6.5 & $>15.8$ & 17.6 & 25 & 13 & 15 \\
\hline 2.0 & 2.55 & 1.71 & 9.3 & 8.1 & $>25.3$ & $>23.3$ & 17.3 & 35.3 & $>23.1$ & 21 & 10 & 11 \\
\hline
\end{tabular}

The evolution of one turn of a spiral emanating from a single dislocation on the (0001) face of $L$-cystine reveals six straight $c / 6$ segments, assigned to $\mathrm{A}(+)$, initially radiating from the core (Figure 3A). Advancement of these steps, which are pinned to the core, results in the formation of new segments associated with $\mathrm{B}(+)$ in the same molecular layer, rotated by $60^{\circ}$ with respect to $\mathrm{A}(+)$ as required by symmetry. Steps with $\mathrm{B}(+)$ orientations increase in length, advancing only when they reach a critical length $L_{c}{ }^{8,30}$ The time elapsed between the onset of straight segment $\mathrm{A}(+)$ (Figure $3 \mathrm{M}$ ) and the onset of the advancement of $\mathrm{B}(+)$ (Figures 3M-P, 3A-F) requires more than one-half of the duration of the entire spiral growth cycle, reflecting the slow velocity of $\mathrm{A}(+)$. This process is followed by fast formation of segments along the $\mathrm{C}(+)$ and $\mathrm{A}(-)$ orientations because of the relatively fast advance of the $\mathrm{B}(+)$ and $\mathrm{C}(+)$ steps, respectively. $\mathrm{B}(+), \mathrm{C}(+)$ and $\mathrm{A}(-)$, all with higher step velocities than $\mathrm{A}(+)$, catch up to $\mathrm{A}(+)$, initially forming a slow-growing bunch consisting of 2-4 steps (Figure 3G). Although six equivalent bunches are anticipated, asymmetry of the core results in the formation of only a few bunched edges initially (Figure 3G), eventually forming six bunched edges (Figure 3I) but with a height of 2-4 elementary layers. At this point, the core appears as it did at the beginning of the cycle (cf. Figure 3I and 3A), although in Figure 3A the pinwheel is formed by A(+) steps while in Figure 3I it is formed by A(-) steps. The slow propagation along $\mathrm{A}(-)$ determines the time required for $\mathrm{B}(-)$ to reach the critical length (Figure $3 \mathrm{H}-\mathrm{L}$ ), after which $\mathrm{B}(-)$ advances faster by comparison, forming segment $\mathrm{C}(-)$ that in turn advances and spawns $\mathrm{A}(+)$. Because segment $\mathrm{A}(+)$ has the slowest velocity it is the orientation most evident in the core region as well as on terraces beyond the core (Figure 8A, below), thereby creating the impression of a pinwheel (Figure 3P). After one complete revolution of the spiral, other segments are terminated either by the spiral center or by the step bunch, which has a height corresponding to six elementary layers. After the generation of multiple spirals a hillock forms that resembles a stack of isolated islands separated by terraces. These islands, however, are not closed loops. Rather, they are a manifestation of the six-threaded spiral coinciding with the crystallographic $6_{1}$ screw axis. ${ }^{29}$ 

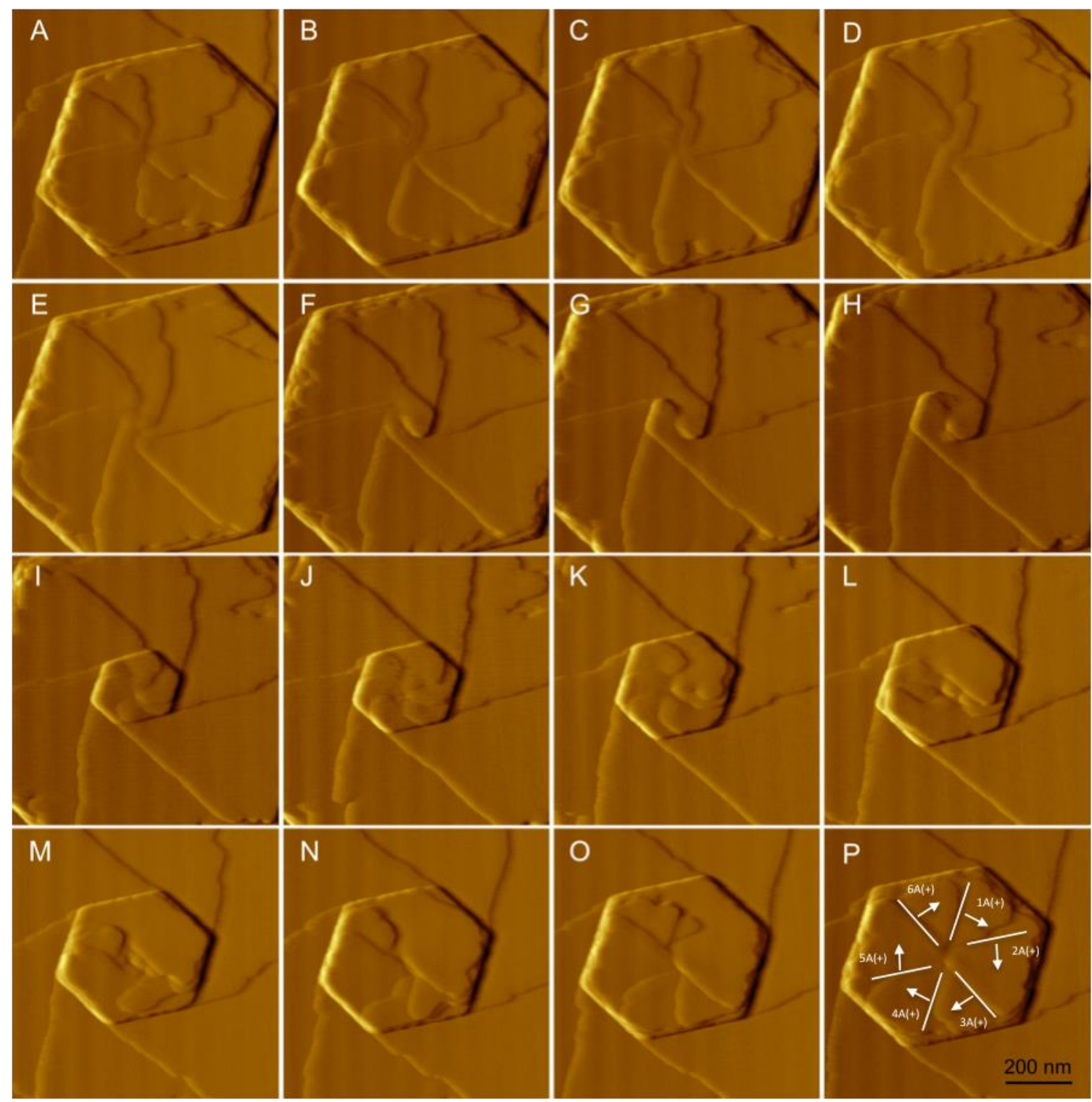

Figure 3. Series of AFM images showing the evolution of the core morphology during one turn of the spiral. The longest step edges are presumed to be $A(+)$. L-cystine concentration $C=1.2 \mathrm{mM}$. The time interval between images is 10.5 seconds.

The slow advancement of step $\mathrm{A}(+)$ enabled measurement of the critical length of the $\mathrm{B}(+)$ step. Measurements of the critical length required for the onset of step advancement were precluded for the remaining faster growing steps by the slow timescale of the AFM measurement. Interestingly, faceted protrusions are often observed on step $\mathrm{B}(+)$ distant from the core, revealing that segment $\mathrm{B}(+)$ near the core is immobile. This is illustrated in Figures $2 \mathrm{~A}-\mathrm{B}$, in which the $\mathrm{B}(+)$ segment reaches a length of 100 $\mathrm{nm}$, after which a protrusion with a step parallel to $\mathrm{B}(+)$ advances at the far end of the segment. The width of the protrusion, $30 \mathrm{~nm}$, can be attributed to an upper limit of the critical length, $L_{c}$, for $\mathrm{B}(+)$. The critical 
length decreases with increasing supersaturation (Table 2). Although the critical lengths for the other five steps could not be measured it is reasonable to suggest that these values would be comparable to or smaller than the value of $L_{c}$ measured for $\mathrm{B}(+)$.

The step edge energy $\gamma_{i}$ can be estimated with equation (1), where $E_{\mathrm{b} i}$ is the binding energy per molecule in a row of molecules attaching to step edge $i, N_{A}$ is Avogadro's number and $a=0.5422 \mathrm{~nm}$, the lattice spacing for $L$-cystine parallel to a $\{10 \overline{10}\}$ step in the $\{0001\}$ plane. The binding energies for steps $\mathrm{A}(+)$ and $\mathrm{A}(-), E_{\mathrm{bA}}=-94 \mathrm{~kJ} / \mathrm{mol}(-22 \mathrm{kcal} / \mathrm{mol})$, are smaller than those for steps $\mathrm{B}(+), \mathrm{B}(-), \mathrm{C}(+)$, and $\mathrm{C}(-), E_{\mathrm{bB}}$ $=E_{\mathrm{bC}}=-152 \mathrm{~kJ} / \mathrm{mol}(-36 \mathrm{kcal} / \mathrm{mol})($ Table 1$)$, corresponding to edge energies of $\gamma_{\mathrm{A}}=1.4 \times 10^{-10} \mathrm{~J} / \mathrm{m}$ and $\gamma_{\mathrm{B}}=\gamma_{\mathrm{C}}=2.3 \times 10^{-10} \mathrm{~J} / \mathrm{m}$, respectively. The step surface energies, $6 \gamma_{i} / c$, calculated from the respective edge energies divided by the height of the step riser $c / 6$, are 0.15 and $0.25 \mathrm{~J} / \mathrm{m}^{2}$. These values are not unlike those reported for similar crystals ${ }^{8,31}$ as well the value $\left(6 / c=0.1 \mathrm{~J} / \mathrm{m}^{2}\right)$ surmised from a correlation between solubility and surface energy for a variety of compounds. ${ }^{32}$

$$
\gamma_{i}=\frac{-E_{\mathrm{b} i}}{2 N_{A} a} ; i=\mathrm{A}, \mathrm{B}, \mathrm{C}
$$

Edge lengths of a hexagonal elementary layer can be deduced by minimizing the sum of the volume and edge energies of a hexagonal nucleus (see Appendix) ${ }^{33}$. In the case of $L$-cystine, the hexagonal nucleus consisting of one elementary layer is characterized by two edge types, A and B, corresponding to the slow $(\mathrm{A}(+)$ and $\mathrm{A}(-))$ and fast $(\mathrm{B}(+), \mathrm{B}(-), \mathrm{C}(+)$, and $\mathrm{C}(-))$ edges. The critical lengths for the slow and fast step edge types, $L_{c \mathrm{~A}}$ and $L_{c \mathrm{~B}}$, depend on the step surface energies and the driving force for crystallization, defined as $\Delta \mu_{0}=\mathrm{R} T \ln \left(C / C_{e q}\right)$, according to equations (2a) and (2b), where $\mathrm{R}$ is the universal gas constant and $T$ is the absolute temperature.

$$
\begin{aligned}
& L_{c \mathrm{~A}}=\frac{\left(2 \gamma_{\mathrm{B}}-\gamma_{\mathrm{A}}\right) N_{A} a^{2}}{\Delta \mu_{0}} \\
& L_{c \mathrm{~B}}=L_{c \mathrm{C}}=\frac{\gamma_{\mathrm{A}} N_{A} a^{2}}{\Delta \mu_{0}}
\end{aligned}
$$

Critical lengths can be calculated from the binding energy per molecule in a row of molecules attaching to a step edge $i, E_{\mathrm{b} i}$, by substitution of eq. (1) into (2b), resulting in eq. (3).

$$
L_{c \mathrm{~B}}=\frac{-E_{\mathrm{bA}} a}{2 \Delta \mu_{0}}
$$


Critical lengths calculated for the fast growing step edges, $L_{c \mathrm{~B}}$, using eq. (3) and $E_{\mathrm{bA}}=-94 \mathrm{~kJ} / \mathrm{mol}(-22$ $\mathrm{kcal} / \mathrm{mol}$ ) (Table 1) were roughly half as large as those measured by AFM at various supersaturations (Table 2). Nonetheless, this correspondence between calculations and measurements is reasonably good given that impurity-induced error in AFM measurements would inflate the measured critical lengths. Unknown impurities in the growth solution can result in step pinning of a developing step edge that would necessitate a longer segment length for the segment to be stable. ${ }^{34}$ Furthermore, discrepancy between the calculated and measured critical lengths can result from errors in the binding energy $E_{\mathrm{bA}}$, that was calculated using implicit solvation and does not mimic solvent-crystal interactions precisely.

Spacing of step bunches on $\{0001\} \boldsymbol{L}$-cystine faces. The spacing of step bunches, $d$, is governed by the slowest step that underlays the bunch, $\mathrm{A}(+)$ in this case. Using a simple geometric construction, an anisotropic polygonal spiral with $N \operatorname{sides}^{35}$ has a characteristic spiral rotation time given by eq. (4), where $L_{c i}$ and $V_{i}$ are the critical length and velocity of the $i^{\text {th }}$ segment, respectively, and $\alpha_{i, i-1}$ is the angle between $i^{\text {th }}$ and $(i-1)^{\text {th }}$ segments of the spiral.

$$
\tau=\sum_{i=1}^{N} \frac{L_{c i} \sin \left(\alpha_{i, i-1}\right)}{V_{i-1}}
$$

The periodic nature of the spiral requires that $\alpha_{N+1, N}=\alpha_{1, N}$. In the case of $L$-cystine, $N=6$, $\sin \left(\alpha_{i, i-1}\right)=\sin (2 \pi / 3)=\sqrt{3} / 2, i=1,2, \ldots, 6$, corresponding to $\mathrm{A}(+), \mathrm{B}(+), \ldots \mathrm{C}(-)$. The spacing of step bunches, $d$, in all six directions is given by eq. (5)

$$
d=V_{\min } \tau=V_{\mathrm{A}(+)} \frac{\sqrt{3}}{2} \sum_{i=1}^{6} \frac{L_{c i}}{V_{i-1}} .
$$

Assuming that all $L_{c i}$ are comparable and substituting measured $L_{c \mathrm{~B}}$ and $V_{i}$ values (Table 2), equation (5) predicts $d$ values (olive squares in Figure 4) that are approximately six times smaller than measured values (black and red triangles in Figure 4). 


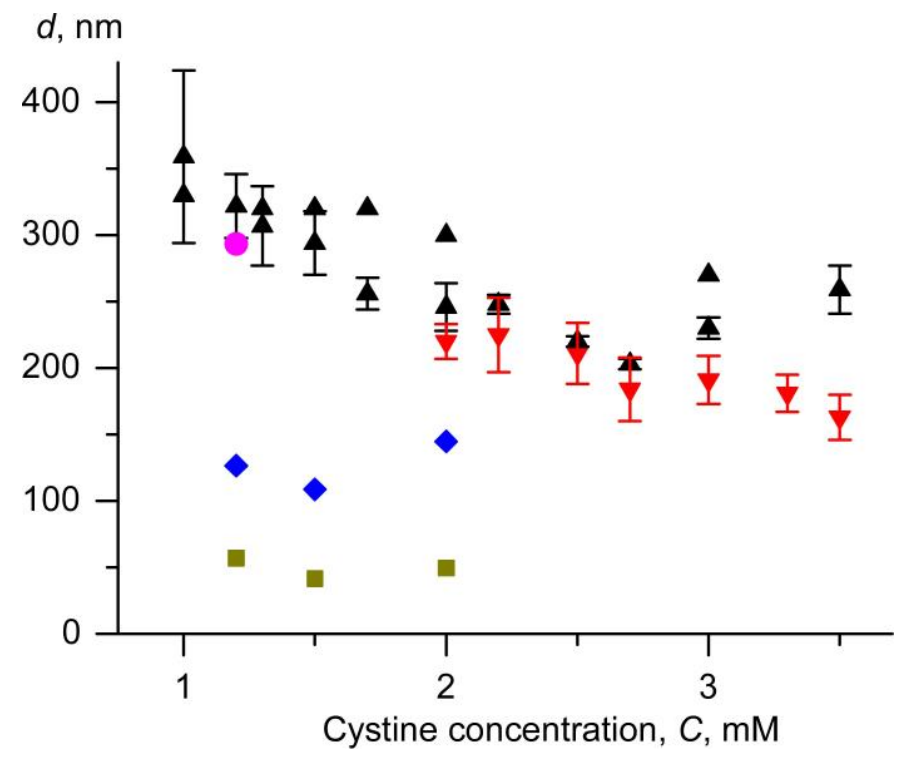

Figure 4. The dependence of step bunch spacings $(d)$ on $L$-cystine concentration $(C)$. Black and red triangles correspond to $C_{\mathrm{CDME}}$ $=0$ and $0.04 \mathrm{mM}$, respectively. The error bars correspond to the standard deviations for measurements averaged over the six crystallographically equivalent $\langle 11 \overline{2} 0\rangle$ directions. Olive squares, blue diamonds, and magenta circles correspond to $d$ calculated from equations (5), (12), and (13), respectively. Radii of the highly strained region used in the calculations: $r_{0}=70 \mathrm{~nm}$ (eq. (12)) (illustrated schematically in Figure 6B), $r_{01}=90 \mathrm{~nm}$ and $r_{02}=40 \mathrm{~nm}$ (eq. (13)) (Figure 5A).

Close inspection of the dislocation core revealed protrusions along step segments distant from the core and immobile step segments in the immediate vicinity of the dislocation (Figures 2B, 5A). Within this region surrounding the dislocation core, steps move slowly and segments of new orientations do not form, resulting in a cavity around the dislocation core with a radius range of $r_{0}=40-90 \mathrm{~nm}$ (Figure 5A,C). The value of $r_{0}$ appeared to decrease with increasing supersaturation, although significant step roughness at low supersaturations and high step velocities at high supersaturations precluded accurate measurements. The size of the cavity oscillates in time along with the stages of spiral formation. At the onset of spiral formation, at which each minor step edge corresponds to $\mathrm{A}(+)$, a well-defined hollow core is observed (Figures 3A,B,P, 5D). The formation of $\mathrm{B}(-), \mathrm{C}(-)$, and $\mathrm{A}(+)$ during the turn of the spiral results in an increase in the size of the cavity surrounding the dislocation core (Figures 3L,M, 5C). The presence of a cavity around the dislocation core suggests the presence of a highly strained region, which would increase the free energy of the crystal in this region and thus decrease the driving force for crystallization, leading to a suppression of growth. The presence of such a highly strained region around the dislocation core could account for the discrepancy between the measured and calculated step bunch spacings. 

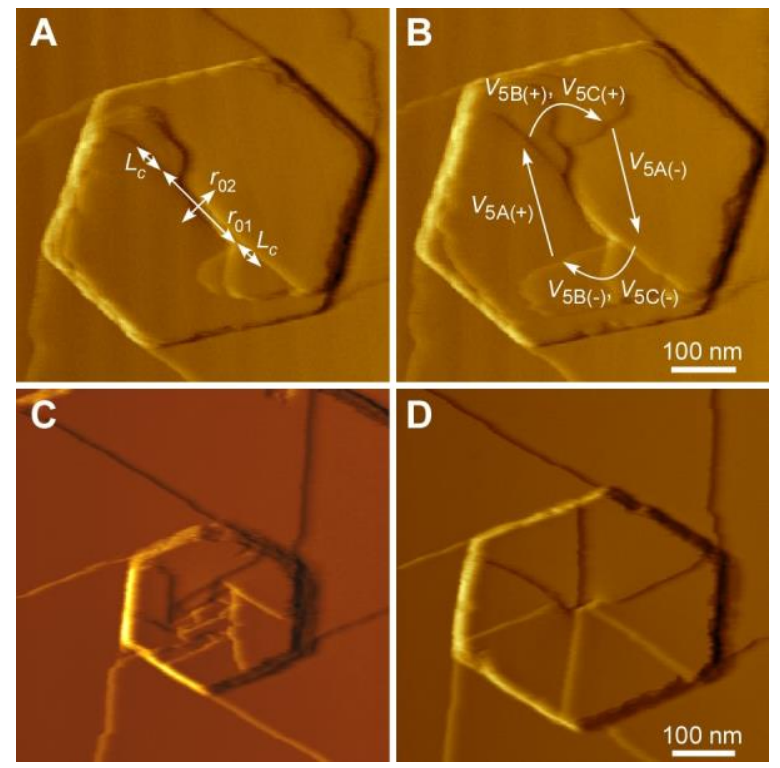

Figure 5. $(\mathrm{A}, \mathrm{B})$ Two subsequent images $(\Delta t=5.3 \mathrm{~s})$ for $L$-cystine growing at $C=1.2 \mathrm{mM}$. Radii $r_{01}$ and $r_{02}$ show anisotropy of growth suppression around the dislocation core. Straight arrows show growth directions for $5 A(+)$ and $5 A(-)$. Curved arrows illustrate fast formation of new segments with rapid change of the step growth direction. Identification of the slowest growing edge in (B) reveals that this is the $5^{\text {th }}$ elementary layer relative to Figure 2 (the $A(+)$ step is parallel to 8 o'clock as opposed to 1 o'clock in Figure 2). (C,D) Two subsequent images $(\Delta t=18.6 \mathrm{~s})$ for $L$-cystine crystal growing at $C=1.5 \mathrm{mM}$. The cavity in image (C) is almost completely gone in image (D), leaving a small hollow core.

The excess elastic energy $U$ associated with the strained core decreases the driving force for crystallization according to eq. (6), where $\Delta \mu_{0}$ is the driving force for crystallization in the absence of a strained core. Impurities reduce the driving force for crystallization by $\Delta \mu_{d}=\mathrm{R} \ln \left(C_{d} / C_{e q}\right)$, where $C_{d}$ is the $L$-cystine concentration above which step velocity is not slowed by impurities, and $C_{e q}$ is the solubility of $L$-cystine in the absence of impurities.

$$
\Delta u=\Delta u_{6}-\Delta u_{d}-U
$$

Excess elastic energy around the dislocation line is given by eq. (7), ${ }^{36}$ where $\Lambda$ is a heat of fusion and $r_{h}$ is the Hooke radius, given by eq. (8). The Burgers vector magnitude $b$ is equal to the lattice constant $c=$ $5.6275 \mathrm{~nm}, \omega$ is molar volume of $L$-cystine $\left(1.42 \cdot 10^{-4} \mathrm{~m}^{3} / \mathrm{mol}\right), s$ is a geometric constant related to the orientation of Burgers vector with respect to the dislocation line ( $s=1$ for a screw dislocation), and $G$ is the shear modulus of $L$-cystine, which corresponds to an elastically isotropic body. ${ }^{37}$ Although the elastic moduli (bulk, Young, and shear) of $L$-cystine kidney stones (aggregates of numerous small crystals) have been reported, ${ }^{37}$ the single-crystal elastic constants of $L$-cystine are not known. Using the Reuss, Voigt, and Hill approximations - averaging schemes by which anisotropic single-crystal elastic constants can be converted into isotropic, polycrystalline elastic moduli - the isotropic, polycrystalline elastic moduli were 
calculated for single-crystalline $L$-cystine using GULP (GAFF forcefield) and Materials Studio (COMPASS forcefield implemented in Forcite). The elastic moduli of kidney stones (25.4, 20.1, and 7.3 $\mathrm{GPa}$, corresponding to the bulk, Young, and shear moduli, respectively) ${ }^{37}$ are somewhat consistent with the calculated moduli (23.9-26.1, 28.3-50.4, and 6.4-13.6 GPa). The calculated and measured bulk and shear moduli match very well, while the calculated Young modulus calculated for a single crystal is larger than the measured polycrystalline sample. This is not surprising because the presence of intercrystalline boundaries should make the material softer, thereby reducing the Young's modulus.

$$
\begin{aligned}
& U=\frac{\Lambda}{1+r^{2} / r_{h}^{2}} \\
& r_{h}=\frac{b}{\pi} \sqrt{\frac{G \omega}{8 s \Lambda}}
\end{aligned}
$$

The radius of the strained screw dislocation core can be calculated from eq. (7) as the radius $r_{V=0}$, at which the driving force for crystallization is zero, $\Delta \mu=0$. Combining expressions (6) $-(8)$, where $r=r_{V=0}$, affords eq. (9).

$$
r_{V=0}=r_{h} \sqrt{\frac{\Lambda}{\Delta \mu_{0}-\Delta \mu_{d}}-1}
$$

For molecular crystals of similar melting points, $T_{m}=230-270^{\circ} \mathrm{C}, \Lambda \sim 24-37 \mathrm{~kJ} / \mathrm{mol}^{38,39}$ and $U=\Delta \mu_{0}-$ $\Delta \mu_{d}=0.45-1.71 \mathrm{~kJ} / \mathrm{mol}$. Therefore, $\Lambda>>\left(\Delta \mu_{0}-\Delta \mu_{d}\right)$, then $\Lambda /\left(\Delta \mu_{0}-\Delta \mu_{d}\right) \gg>1$, and the unknown $\Lambda$ can be eliminated from eq. (9).

Eq. (8) was derived assuming elastic isotropy of the crystal, however, for screw dislocations in hexagonal crystals with Burgers vector oriented along [0001], the elastic stiffness constant $c_{44}$ should be used instead of the isotropic $G .^{40}$ The elastic stiffness tensors, $c_{i j}$, were calculated for $L$-cystine using two methods (Table 3), which afforded $c_{44}=3.1 \mathrm{GPa}$ (GULP, GAFF forcefield) and $c_{44}=4.2 \mathrm{GPa}$ (Forcite, COMPASS forcefield) (Table 3).

Dislocation-actuated Growth and Inhibition of Hexagonal L-cystine Crystallization at the Molecular Level 
Table 3. Calculated elastic stiffness ( $\left.c_{i j}, \mathrm{GPa}\right)$ constants for $L$-cystine. Elastic compliance $\left(s_{i j}, 1 / \mathrm{TPa}\right)$ constants are included for reference.

\begin{tabular}{|c|c|c|c|c|}
\hline \multirow[b]{2}{*}{$i j$} & \multicolumn{2}{|c|}{$\begin{array}{c}\text { GULP, } \\
\text { GAFF } \\
\text { forcefield }\end{array}$} & \multicolumn{2}{|c|}{$\begin{array}{c}\text { Material Studio, } \\
\text { COMPASS } \\
\text { forcefield }\end{array}$} \\
\hline & $c_{i j}$ & $s_{i j}$ & $c_{i j}$ & $s_{i j}$ \\
\hline 11 & 58.3 & 19.8 & 47.3 & 35.3 \\
\hline 12 & 20.8 & -6.9 & 29.5 & -20.8 \\
\hline 13 & 6.7 & -1.8 & 11.6 & -4.9 \\
\hline 33 & 46.7 & 21.9 & 34.3 & 32.4 \\
\hline 44 & 3.1 & 318.6 & 4.19 & 238.8 \\
\hline
\end{tabular}

Replacing Young's modulus in eq. (9) with the stiffness constant, $G=c_{44}$, and substituting the geometric factor for screw dislocations, $s=1$, yields eq. (10). Substituting the stiffness constant, $c_{44}=3.7 \mathrm{GPa}$ (the average of the two calculated values), eq. (10) affords $r_{V=0}=11-22 \mathrm{~nm}$ (Table 2), which is smaller than experimentally measured $r_{0}=40-90 \mathrm{~nm}$ (Figure 5A-C). This discrepancy can arise in part from underestimation of $c_{44}$ (there is no experimental data) and $b$ (possible existence of edge component of the Burgers vector). Additionally, the structure of the core seems to be more complex than was assumed in the derivation of eq. (7) and (8), evident by the observation of elongated cores that do not conform to hexagonal symmetry (Figures 2, 3).

$$
r_{V=0}=\frac{b}{\pi} \sqrt{\frac{c_{44} \omega}{8\left(\Delta \mu_{0}-\Delta \mu_{d}\right)}}
$$

To account for the effect of the strained core region on the step bunch spacing, $d$, equation (5) can be modified such that the time required to create a new segment $i$ is determined not only by the time to create a segment of critical length, $L_{c i}$, but also by the time necessary to traverse a part of the core region perimeter so that creation of the new segment becomes possible (i.e. the time necessary for $L_{c 2}$ to traverse a distance of $d_{12}$ before formation of $L_{c 3}$ in Figure 6). A similar explanation has been invoked for triangular spirals on $\{101\}$ faces of potassium dihydrogen phosphate crystals that surround highly strained dislocation cores. ${ }^{41}$ Adapting this model for $N$-sided spirals with circular cores, Figure 6 affords the time, $\tau_{2}=d_{2} / V_{2}$, between the creation of critical segments of lengths $L_{c 2}$ and $L_{c 3}$. During $\tau_{2}$, segment 2 will travel a distance $d_{2}=d_{12}+d_{c 3}$ with velocity $V_{2}$. The first term, which is equivalent to $d_{12}=r_{0}\left(\cos \alpha_{1,2}\right.$ +1 ) determines the distance traveled by $L_{c 2}$ to traverse the perimeter of a core of radius $r_{0}$ before the segment $L_{c 3}$ can begin to form. The second term equivalent to $d_{c 3}=L_{c 3} \sin \alpha_{2,3}$ refers to the distance traveled by the segment 2 to create a segment 3 of a critical length. The total time for a complete turn of a 
spiral is the sum of all partial times $\tau_{I}$, affording eq. (11). In the specific case of $L$-cystine, for which $N=$ 6 and $\alpha_{i, i-1}=120^{\circ}$, the step bunch spacing is given by eq. (12).

$$
\begin{gathered}
\tau_{s 1}=\sum_{i=1}^{N} \frac{L_{c i} \sin \left(\alpha_{i, i-1}\right)+r_{0}\left(\cos \left(\alpha_{i-1, i-2}\right)+1\right)}{V_{i-1}} \\
d_{s 1}=V_{\min } \tau_{s 1}=\frac{V_{\mathrm{A}(+)}\left(\sqrt{3} L_{c}+3 r_{0}\right) \sum_{i=1}^{6} \frac{1}{V_{i}}}{2}
\end{gathered}
$$

A
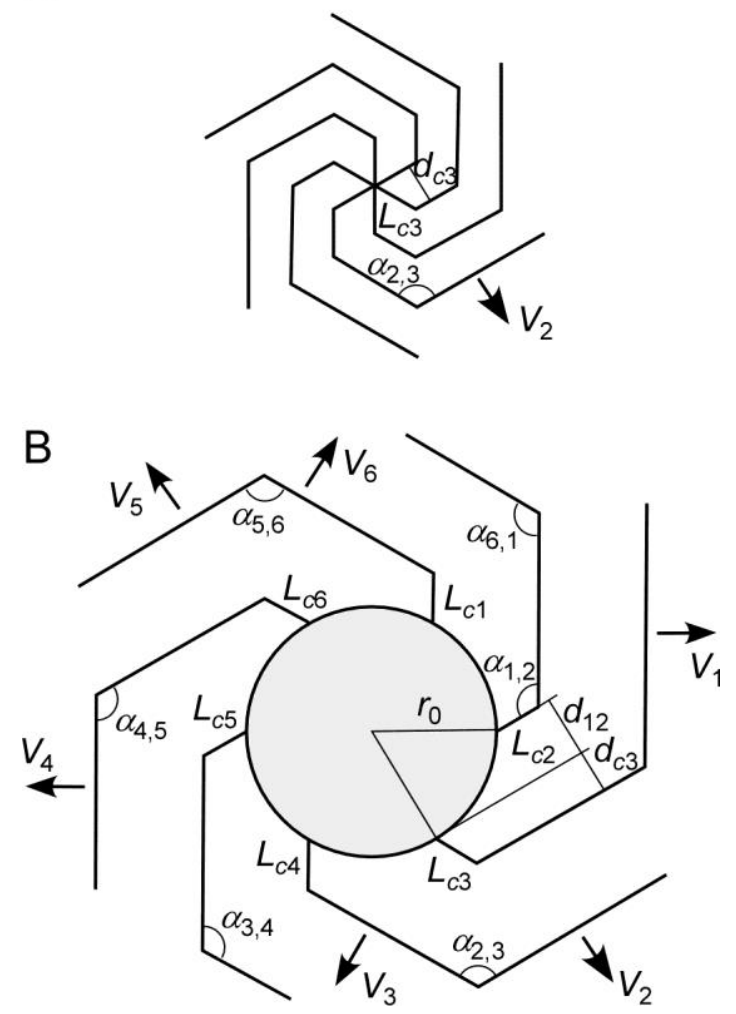

Figure 6. Formation of isotropic hexagonal spiral (A) without and (B) with an inactive (i.e. highly strained) central core of radius $r_{0}$, where growth is suppressed. (A) Segment $L_{c 3}$ is formed after a distance of $d_{c 3}$ is traversed in the absence of a strained dislocation core. (B) Segment $L_{c 3}$ is formed after a distance of $d_{12}+d_{c 3}$ is traversed in the presence of a strained dislocation core.

Step bunch spacings predicted by Equation (12) (blue diamonds in Figure 4) more closely match the observed values of step bunch spacings. A discrepancy remains, however, that can be attributed to an elongated rather than a circular dislocation core. One cycle of the elongated spiral core in Figure 5A can be divided into four parts (Figure 5A,B): 
(i) Slow growth of a step in orientation $\mathrm{A}(+)$ with a distance of $2 r_{01} \cdot \sin (\pi / 3)$, resulting in the formation of the immobile segment $\mathrm{B}(+)$. Propagation of the $\mathrm{B}(+)$ begins when $\mathrm{A}(+)$ travels an additional distance of $L_{c} \cdot \sin (\pi / 3)$;

(ii) Fast growth of $\mathrm{B}(+)$ with fast formation of $\mathrm{C}(+)$ and $\mathrm{A}(-)$ instantaneously thereafter. These segments travel only a distance of approximately $2 r_{02} \cdot \sin (\pi / 3)$, thus defining the ellipticity of the core region. The critical lengths $L_{c(+)}$ and $L_{c \mathrm{~A}(-)}$ cannot be measured, but they are embedded in the distance $2 r_{02} \cdot \sin (\pi / 3)$;

(iii) Slow displacement of $\mathrm{A}(-)$ by a distance of $\left(2 r_{01}+L_{c}\right) \cdot \sin (\pi / 3)$;

(iv) Fast formation of $\mathrm{B}(-), \mathrm{C}(-)$, and $\mathrm{A}(+)$, where segments $\mathrm{B}(-)$ and $\mathrm{C}(-)$ travel a distance of $2 r_{02} \cdot \sin (\pi / 3)$.

Summing the distances traversed around each part of the dislocation core affords eq. (13), which now anticipates step bunch spacings that agree well with the observed values (magenta circle in Figure 4). That is, by accounting for elastic stress around the dislocation line, manifested as suppressed growth in the dislocation outcrop defined by $r_{01}=90 \mathrm{~nm}$ and $r_{02}=40 \mathrm{~nm}$ (see Figure 5A), calculated step bunch spacings are in agreement with measured values. The dislocation core is anisotropic and the step movement around the core follows this anisotropy, further increasing step bunch spacings.

$$
d_{s 2}=\sqrt{\beta}\left[\left(r_{01}+\frac{L_{c \mathrm{~B}}}{2}\right)\left(1+\frac{V_{\mathrm{A}(+)}}{V_{\mathrm{A}(-)}}\right)+r_{02} V_{\mathrm{A}(+)} \sum_{i=\mathrm{B}(+), \mathrm{C}(+), \mathrm{B}(-), \mathrm{C}(-)} \frac{1}{V_{i}}\right]
$$

Effect of CDME on $\boldsymbol{L}$-cystine growth. The presence of relatively small concentrations of the additive $L$ cystine dimethylester (CDME) can reduce step velocities on (0001) $L$-cystine faces substantially. ${ }^{4}$ Figure 7 illustrates the effect of $L$-cystine concentration, $C$, on the velocity of step bunches, $V_{\mathrm{A}}{ }^{*}$, without additives and at various concentrations of CDME. $L$-cystine in the absence of CDME exhibits a "dead zone", characterized by the upper limit concentration of $L$-cystine, $C_{t d}$, which corresponds to the minimum supersaturation required for non-zero step velocity. In the absence of CDME, $L$-cystine growth exhibits a dead zone below $C_{t d}=0.8 \mathrm{mM}$, signifying the presence of an adventitious impurity that inhibits growth. It is likely that the lower concentration limit at which the growth rate is zero corresponds to the solubility of $L$-cystine, $C_{e q}$, which agrees well with published $L$-cystine solubility, $C_{e q}=0.7 \mathrm{mM}^{12-14}$ (with $C_{\mathrm{CDME}}=0 \mathrm{mM}$ ). Dead zones also have been observed for dissolution ${ }^{42}$ and melting, ${ }^{43}$ however; therefore, it can only be stated that solubility of $L$-cystine is within the concentration range of the dead zone. 
The addition of CDME to supersaturated $L$-cystine solutions, such that $\mathrm{C}_{\mathrm{CDME}}=0.056$ and $0.149 \mathrm{mM}$, shifts the upper limit of the dead zone to $C_{t d}=1.1$ and $1.6 \mathrm{mM}$, respectively (Figure 7B). In the presence of CDME, the dead zone is shifted to higher concentration values indicating an effective increase in $L$ cystine solubility.

Above $C_{t d}$, step velocities increase slowly until reaching $C_{d}$, a threshold value above which step velocity increases quickly and linearly. The existence of two threshold values, for the absence of growth $\left(C_{t d}\right)$ and for very slow growth $\left(C_{d}\right)$, respectively, is well known for different compounds crystallizing in the presence of impurities. ${ }^{44-46}$ The slowly increasing step velocities in the range $C_{t d}<C<C_{d}$, indicate an intermediate regime between complete and negligible inhibition of growth by impurities. The upper limit of this range is $C_{d} \approx 1.0,2.0$, and $2.7 \mathrm{mM}$ for $C_{\mathrm{CDME}}=0,0.056$, and $0.149 \mathrm{mM}$, respectively. At $C>C_{d}$ step velocity increases steeply and linearly (Figure 7). The slope, which is proportional to the kinetic coefficient, $\beta$, is nearly identical for $C_{\mathrm{CDME}}=0,0.056$, and $0.149 \mathrm{mM}$.

The addition of CDME to supersaturated $L$-cystine solutions also results in roughened step edges (Figure $8 \mathrm{~A}, \mathrm{~B}$ ), accompanied by a strong linear dependence of $V / V_{\text {ideal }}$, and roughness $R_{0} / R$ (Figure $8 \mathrm{C}$ ) on CDME concentration, where $V_{\text {ideal }}$ is the ideal step velocity at the same supersaturation without added CDME, determined from a linear curve with the same slope but extrapolated from the reported value of $C_{e q}=0.7$ $\mathrm{mM}$; that is, assuming the same kinetic coefficient and the absence of a dead zone. The value of $R_{0}=2.8$ $\mathrm{nm}$ corresponds to the roughness measured for $L$-cystine without $\mathrm{CDME}$ at $C=3.5 \mathrm{mM}$. 
A

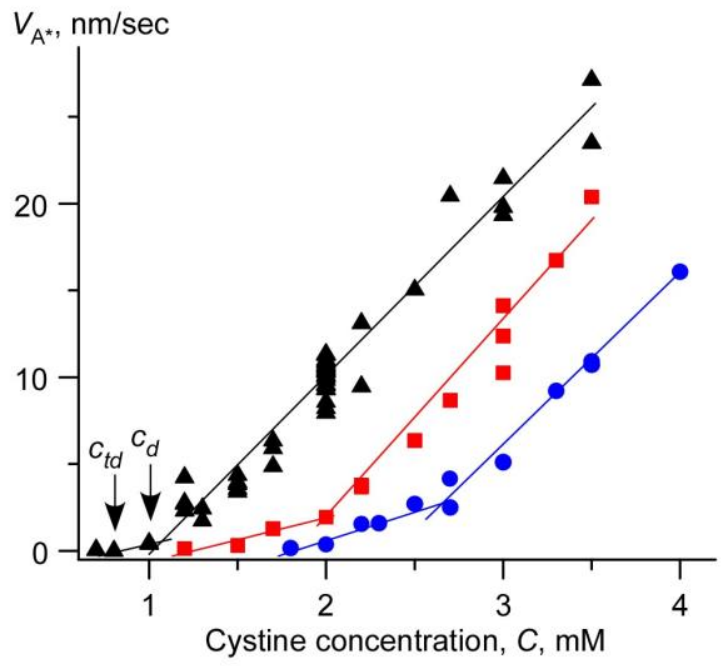

B

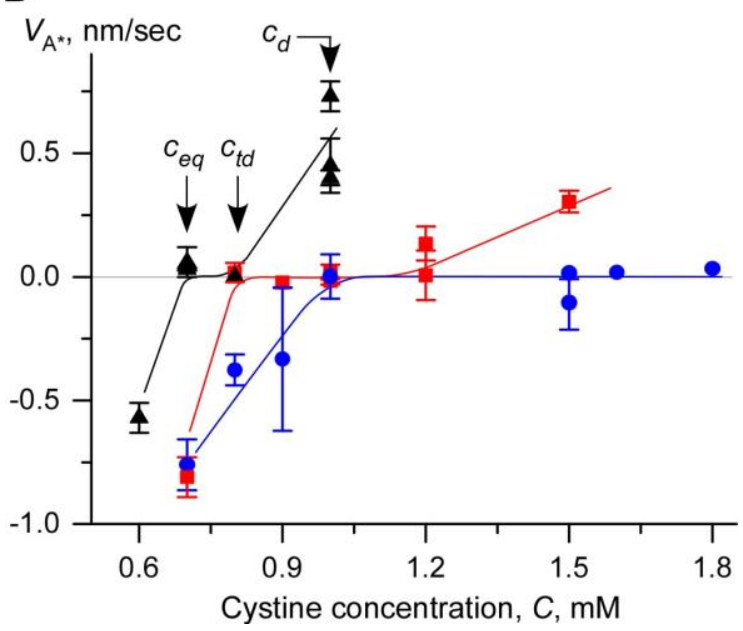

Figure 7. Step velocity $V_{\mathrm{A}^{*}}$ measured for bunches of six steps in $\mathrm{A}(+)$ orientation far from the dislocation outcrop as a function of $L$-cystine concentration in a solution. Black triangles $-C_{\mathrm{CDME}}=0$; red squares $-C_{\mathrm{CDME}}=0.04 \mathrm{mM}$; blue circles $-C_{\mathrm{CDME}}=0.117 \mathrm{mM}$. (A) Step velocities at $L$-cystine concentrations, $C$, ranging from $0-4 \mathrm{mM}$ at different $C_{\mathrm{CDME}}$. The absence of growth at positive $C$ results in a dead zone, the upper limit of which is characterized by $C_{t d}$. Step velocities increase slowly with increasing $C$ until $C_{d}$, after which the step velocities increase quickly and linearly with increasing $C$. The slope is proportional to the kinetic coefficient, $\beta$, and is nearly identical for $C_{\mathrm{CDME}}=0,0.056$, and $0.149 \mathrm{mM}$. For $L$-cystine without additives $\beta=V_{\mathrm{A}}^{*} /\left[\left(C-C_{\text {eq }}\right) \omega\right]=7.0 \cdot 10^{-3} \mathrm{~cm} / \mathrm{s}$. (B) Step velocities in vicinity of the dead zone. Negative step velocities suggest dissolution. Error bars are not shown if they are smaller than the symbol sizes. Some points close to $V=0$ were omitted in Figure 7A. 

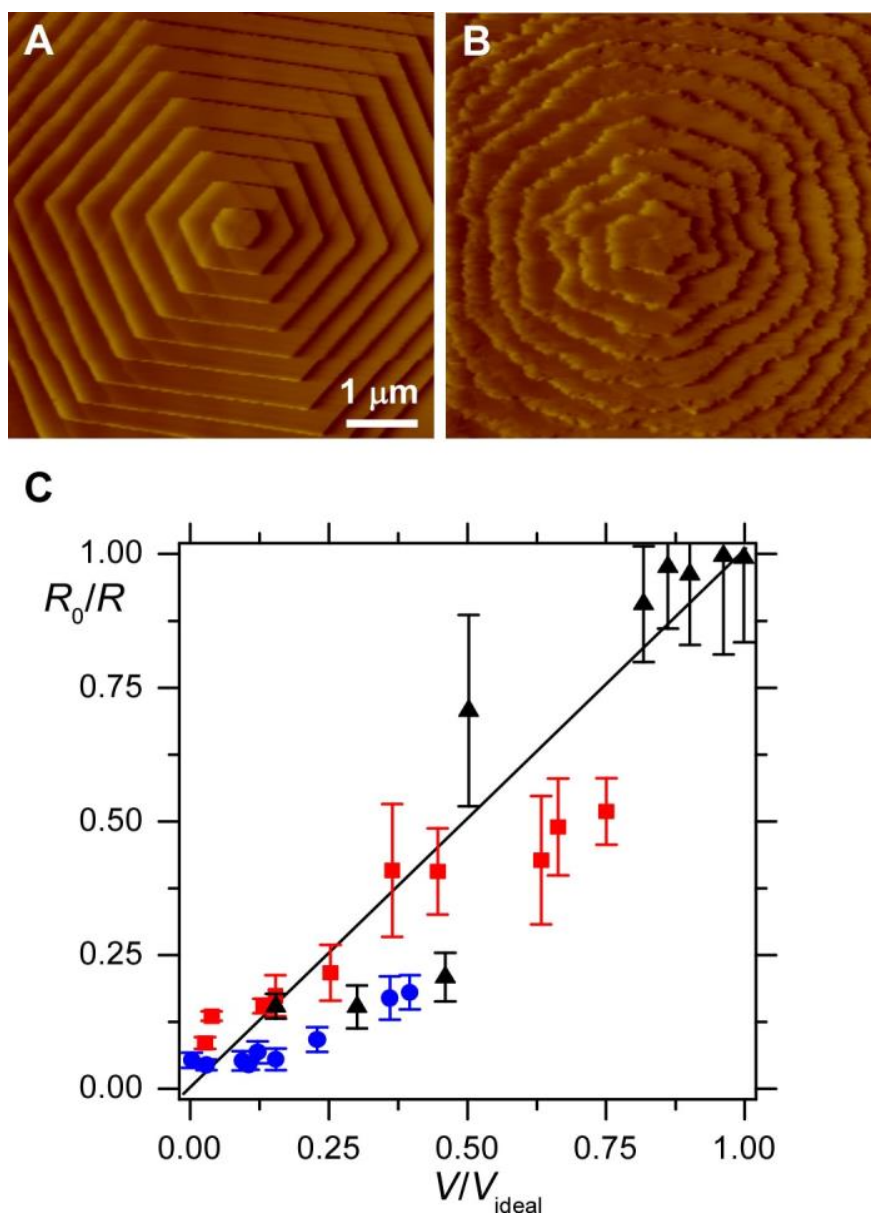

Figure 8. (A,B) $L$-cystine growth hillocks forming at $C=2 \mathrm{mM}$. Step bunch edge roughness is much weaker for impurity free solution (A) compared to solution containing $C_{\mathrm{CDME}}=0.04 \mathrm{mM}(\mathrm{B})$. (C) Relative roughness, $R_{0} / R$, plotted versus relative step velocity, $V / V_{\text {ideal. }}$. Black triangles: $C_{\mathrm{CDME}}=0$; red squares: $C_{\mathrm{CDME}}=0.04 \mathrm{mM}$; blue circles: $C_{\mathrm{CDME}}=0.117 \mathrm{mM}$.

These observed effects of CDME are consistent with the Cabrera-Vermilyea mechanism of impurity action, ${ }^{8,47}$ in which adsorbed impurity particles block step propagation. Although steps can grow past impurity particles, step pinning results in an increased step curvature that decreases the effective supersaturation, as described by the Gibbs-Thomson law. ${ }^{8}$ Because step velocity is proportional to supersaturation, step propagation slows. If the distance between adsorbed impurity particles is smaller than the diameter of the critical nucleus determined by the Gibbs-Thomson law at a particular supersaturation, step curvature is sufficiently high to decrease the effective supersaturation so that growth ceases. This is observed as a shift of the dead zone to higher $L$-cystine supersaturation ranges with increasing concentration of CDME.

The velocity of the step bunch in the presence of impurities is given by eq. (14), ${ }^{47}$ where $V_{0}$ is the step bunch velocity in the absence of added impurities and the diameter of the critical nucleus, $2 r_{c}=L_{c}$, is 
given by eq. (2). The distance between impurity particles $h=a \sqrt{ } \theta$ depends on the unit cell dimension, $a$, and the surface coverage by the impurity, $\theta$, calculated from Henry's adsorption isotherm $\theta=K C_{\text {impurity. }}$ Here $K$ is the adsorption constant and $C_{\text {impurity }}$ is the concentration of impurities. Breaking through the "impurity fence" forces the step velocity to vary from $V_{0}$ (straight step) to $V_{0}\left(1-2 r_{c} / h\right.$ ) (step with maximum curvature). The average step velocity is estimated as a geometric mean of these two extremes.

$$
V=\left\{\begin{array}{cc}
V_{0} \sqrt{1-\frac{2 r_{c}}{h}} & h>2 r_{c} \\
0 & h \leq 2 r_{c}
\end{array}\right.
$$

Equation (14) does not agree well with step velocities of $L$-cystine measured in the presence of CDME, however. The step velocities initially decrease quickly with increasing CDME concentration, $C_{\mathrm{CDME}}$, but this decrease slows at $C_{\mathrm{CDME}} \approx 0.05 \mathrm{mM}$, resulting in a non-zero $V$ for a wide range of $C_{\mathrm{CDME}}$ (Figure 9). Deviations from the classical model described by eq. (14) are not uncommon ${ }^{8,9}$ when growth inhibition occurs via step pinning. ${ }^{44-46,48-52}$ Several modifications to this step velocity model have been proposed, including:

(i) Replacement of Henry's adsorption isotherm with a Langmuir isotherm, ${ }^{50,51,53}$ in which the amount of surface adsorbate depends on the balance between adsorption and desorption processes rather than just proportional to the adsorbate concentration in a solution. This correction can be important for significant surface coverage by the impurity; however, the observation of non-zero step velocities of $L$-cystine in the presence of CDME is consistent with low surface coverage by strongly-adsorbed CDME stoppers.

(ii) Application of a more correct expression for the driving force of crystallization $(V \sim \Delta C$ instead of $V \sim \Delta \mu)^{46}$ is important if $C \gg C_{e q}$ and the approximation $\Delta \mu=\mathrm{R} T \ln \left(C / C_{e q}\right) \approx \mathrm{R} T(C$ - $\left.C_{e q}\right) / C_{e q}$ no longer holds. For $L$-cystine, however, $C$ is close to $C_{e q}$ and the difference is small.

(iii) A better fit between calculated and measured step velocities has been reported using the arithmetic mean ${ }^{54}$ or minimum velocities ${ }^{50,51}$ instead of the geometric mean. These corrections, however, are not very well justified. The use of minimum step velocities slightly modifies the predicted step velocities but does not resolve the discrepancy between the calculated and measured values.

(iv) The Gibbs-Thomson law serves as the basis for the inhibition mechanism. It is not applicable for systems with small kink densities. ${ }^{9,55}$ For $L$-cystine, this possibility is unlikely because 
measured critical lengths are short (21-33 nm or 40-60 unit cells, Table 2) and in the presence of impurities steps on the (0001) L-cystine surface become rounded and rough, suggesting high kink densities for steps propagating along all $\{1010\}$ orientations. Moreover, compared with crystals having low kink density, $L$-cystine is characterized by relatively high solubility and growth rate.

(v) Impurities play a dual role not only pinning steps, but also slowing down attachment to kinks (combination of Cabrera-Vermilyea and Bliznakov-Chernov mechanisms). ${ }^{56}$ The dual role of impurities is confirmed by the direct dependence of dead zone width (a feature attributable to the Cabrera-Vermilyea step pinning mechanism) and the inverse dependence of kinetic coefficient (a feature attributable to the Bliznakov-Chernov kink blocking mechanism) with impurity concentration. ${ }^{50,51}$ For $L$-cystine, however, the kinetic coefficient does not change significantly with increasing concentration of CDME (Figure 7), indicating that the BliznakovChernov kink blocking mechanism is not applicable to $L$-cystine.

(vi) Generally, elementary steps and macrosteps consisting of many elementary steps show different mobilities for the same impurity concentration. Macrosteps, then, might be responsible for the deviation from classical behavior. ${ }^{45,49}$ But for $L$-cystine, the only step configuration observed far from dislocation outcrops is a single bunch consisting of six elementary steps.

(vii) If the characteristic time of impurity adsorption is comparable or larger than the terrace exposure time, the surface impurity concentration can show a complicated dependence on the step velocity. ${ }^{52,57,58}$ Comparable adsorption times for similarly sized $L$-cystine and CDME molecules are expected, however.

(viii) Impurity "stoppers" can have a finite lifetime ${ }^{45}$ because they can detach from the surface or be incorporated by advancing steps. If $h>2 r_{c}$, impurity adsorption/desorption processes do not have significant effects on the step velocity, but as $h$ approaches $2 r_{c}$ the step velocity will be controlled by the lifetime of stoppers. If this lifetime is not large, the decrease in step velocity with increasing impurity concentration will be much more gradual than if stoppers are absolutely immobile.

(ix) The stochastic impurity distribution over the crystal surface can result in different regimes of step advancement for $h>2 r_{c}$ and $h \approx 2 r_{c} .{ }^{44,59}$ As a result, step velocity does not approach zero

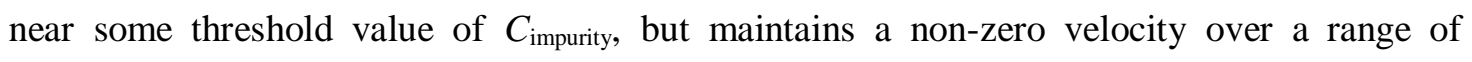
impurity concentrations. 
The two latter modifications to the step velocity model described by eq. (14) are applicable to a wide variety of crystals, including $L$-cystine, but the verification of these modifications is restricted by the absence of a quantitative theory.

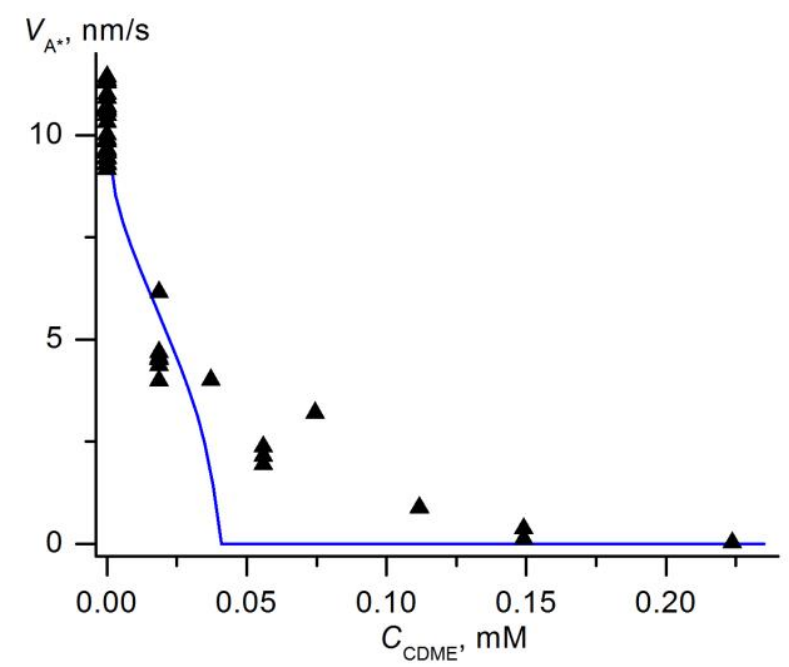

Figure 9. Step velocity dependence of the six-step bunches along the $A(+)$ orientation on CDME concentration, distant from the dislocation core. The triangles correspond to experimental data and the blue curve corresponds to the classic CabreraVermilyea model, equation (14), with $K=6.8 \mathrm{~L} / \mathrm{mol}$. $C=2 \mathrm{mM}$.

Mechanism of CDME incorporation into $L$-cystine crystals. The simple kinetic consideration discussed above does not elucidate the mechanism of CDME interaction with $L$-cystine during crystallization from solution. It does not distinguish whether CDME forms a solid solution with $L$-cystine, adsorbs on the $L$-cystine crystal surface, becomes incorporated into the crystal structure in the form of randomly oriented molecular clusters, or is not captured by the crystal at all. NMR measurements revealed that the total CDME concentration in $L$-cystine crystals grown in $3 \mathrm{mM} L$-cystine solutions containing 0.015 mM CDME is $0.28-0.45 \mathrm{~mol} \%$ (0.39 mol \% if averaged over all four measurements). These data do not reveal the distribution of CDME in the crystal, however.

Hexagonal $L$-cystine plates should be optically uniaxial. If viewed between crossed polarizers with the unique axis parallel to the light path, the crystals should be extinguished in all azimuthal orientations of the crystal with respect to the crossed polarizer and analyzer. ${ }^{60,61} L$-cystine crystals grown in the absence of CDME, however, were slightly biaxial with $\Delta n \approx 1.3 \cdot 10^{-4}$. The orientation of the fast and slow axes in anomalously birefringent $\{10 \overline{10}\}$ growth sectors followed the hexagonal symmetry (Figure 10A-C). The orientation of the optical indicatrix is uniform within each $\{1010\}$ growth sector. The larger refractive index is oriented along each of the $\{1010\}$ faces, and consequently the larger refractive index is parallel to 
the respective growth fronts (Figure 10A,B). This observation suggests the incorporation of an adventitious impurity into the $L$-cystine crystal, ${ }^{61,62}$ consistent with the kinetic dead zone observed for ostensibly pure $L$-cystine. Moreover, the impurity must be oriented identically in each sector with respect to the hexagonal symmetry of the crystal. Crystals grown in solutions containing small amounts of $\operatorname{CDME}\left(C_{\mathrm{CDME}}=0.006 \mathrm{mM}\right)$ exhibited only a slightly larger anomalous birefringence, ca. $1.4 \cdot 10^{-4}$, but the birefringence became significantly larger at $C_{\mathrm{CDME}}=0.015 \mathrm{mM}$, ranging from $4 \cdot 10^{-4}$ to $7 \cdot 10^{-4}$ (Figure 10D-F). Observations at higher CDME concentrations were not possible because the resulting hexagonal crystals were very elongated along [0001] and the tetragonal $L$-cystine polymorph was formed preferentially. The $\{1010\}$ sector-zoned distribution of anomalous birefringence suggests that the optical desymmetrization is a result of CDME incorporation into the $L$-cystine crystal lattice. The relative concentrations of CDME in $\{0001\}$ and $\{10 \overline{10}\}$ growth sectors are not known (Figure 10), but a higher CDME concentration in the $\{10 \overline{10}\}$ growth sectors can be presumed given that the growth rate normal to the $\{1010\}$ faces (i.e. the step bunches) decreases much more (ca. 50 times) than that of the $\{0001\}$ faces (ca. 2 times) as CDME concentration in solution is increased from 0 to $0.015 \mathrm{mM}$. Diminished retardance at the boundary between growth sectors (e.g. blue spokes in Figure 10F) may be a consequence of overlapping of adjacent sectors along the light path or compensatory stress birefringence at these boundaries. 

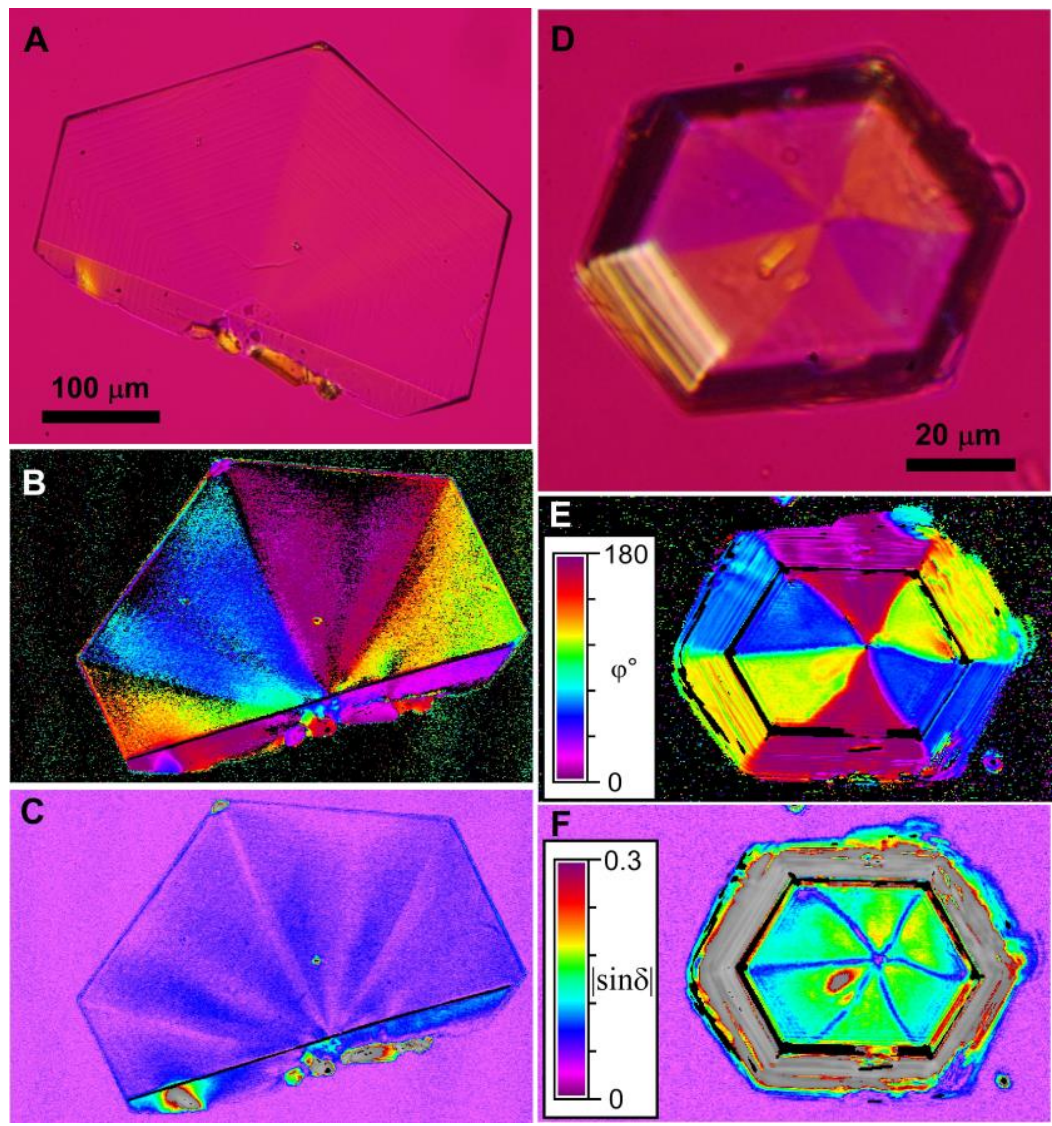

Figure 10. Anomalous birefringence in pure $L$-cystine crystals $(A-C)$ and crystals with $0.015 \mathrm{mM} C D M E$ (D-F) viewed along the [0001] axis. $C=3 \mathrm{mM}$. (A,D) Polarized light micrographs; crossed polarizers are oriented vertically and horizontally; first order red retarder is inserted. $(B, C)$ and $(E, F)$ Birefringence imaging with red light, $\lambda=630 \mathrm{~nm}$, for two crystals. (B,E) False color map of the orientation $\left(\varphi,{ }^{\circ}\right)$ of the larger refractive index, $N_{z}^{\prime}$, in the cross section measured counterclockwise from horizontal axis (scale inset in (E) is same for (B)). (C,F) Retardance $(\delta$, rad) is mapped as absolute value of $\mid$ sin $\delta \mid$ using false color scale (shown in the inset; the same for both images). Grey color indicates that retardance could not be calculated from the data as the transmitted intensity through the inclined faces was too small). Birefringence is calculated as $\Delta n=\lambda \delta /(2 \pi H)$, where $H$ is the thickness of the sample.

The molecular origin of the anomalous birefringence can be rationalized on the basis of bond refractivity in the $L$-cystine crystal structure admixed with CDME. The esterification of terminal hydroxyl groups, resulting in the substitution of $-\mathrm{CH}_{3}$ for the terminal $-\mathrm{H}$ atoms on $L$-cystine, is expected to produce only a small perturbation in the optical indicatrix because the refractivity of $\mathrm{O}-\mathrm{H}$ and $\mathrm{O}-\mathrm{C}$ bonds are comparable (1.8 and $1.5 \mathrm{~cm}^{3} / \mathrm{mol}$, respectively ${ }^{62}$ ). The refractivity of the S-S bond, however, is much larger (ca. 8 $\left.\mathrm{cm}^{3} / \mathrm{mol}\right){ }^{62}$ In the ideal hexagonal $L$-cystine structure all S-S bonds lie in $\{1010\}$ planes and nearly parallel to $<11 \overline{2} 0>$ directions (Figure 1B). Due to the presence of the $6_{1}$ screw axis, the orientation of the $\mathrm{S}-\mathrm{S}$ bond in (0001) plane rotates in $60^{\circ}$ increments from layer $\mathrm{C} 1$ to $\mathrm{C6}$ (Figure 1A), such that the average projection of all S-S bonds onto the (0001) plane equals zero. 
Changes in the average S-S bond orientation that may be attributed to the increased birefringence upon CDME incorporation in $L$-cystine crystals were explored through simulations of a mixed crystal wherein an $L$-cystine molecule in the center of a $5 a \times 4 b \times 1 c$ supercell was replaced with a CDME molecule, and the crystal structure optimized in the region immediately surrounding the CDME molecule. The resulting structure revealed a distortion from the parent $L$-cystine crystal structure, wherein the vector sum of the projections of all S-S bonds onto the (0001) plane in the crystal structure became nonzero, as expected. Notably, the vector sum of the S-S bonds for the entire supercell was oriented along only one of the six \{ 1010 \} planes, nearly coinciding with the orientation of the S-S bond of the replaced $L$-cystine molecule. The birefringence measurements demonstrated that the larger refractive index $N_{Z}$ in crystals with incorporated CDME was parallel to the $\{10 \overline{10}\}$ faces (Figure 10E) in every $\{10 \overline{10}\}$ growth sector, consistent with the S-S bond vector parallel to these faces. Combined with the computational result, the observed birefringence argues that CDME is preferentially incorporated during crystal growth at steps corresponding to $\mathrm{A}(+)$ or $\mathrm{A}(-)$. Calculation of the stereospecific replacement energies for CDME, however, revealed a trend that suggested CDME incorporation is favored at $\mathrm{A}(+)$ (Table 4). This conclusion also agrees well with the proposed mode of CDME binding to $L$-cystine, ${ }^{4}$ where preferential attachment of CDME to one of the six crystallographically unique sites on each of the $\{1010\}$ faces was invoked on the basis of simple models and crystal morphologies. Collectively, these observations indicate that CDME is incorporated in $L$-cystine with remarkable fidelity with respect to its orientation at the growing steps.

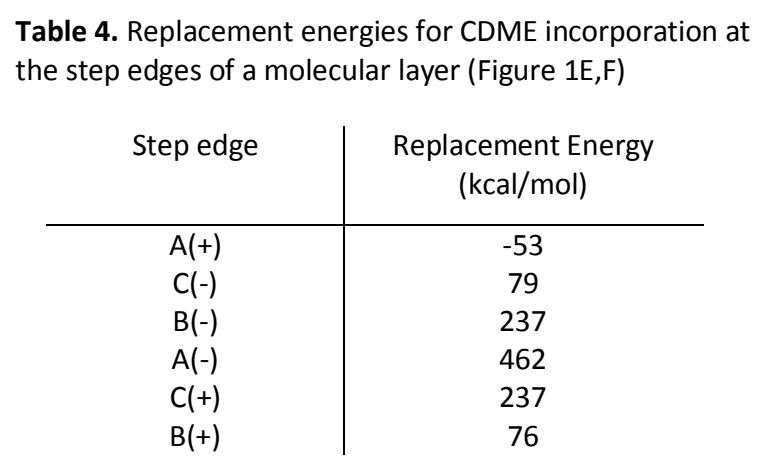

\section{CONCLUSIONS}

Dislocation spirals on the (0001) face of hexagonal $L$-cystine beautifully illustrate the complexity of crystal growth and the correspondence between crystal symmetry and microscopic features, herein evident from real-time in situ AFM. The presence of six equivalent layers of $L$-cystine molecules within one unit cell, wherein each layer is characterized by six crystallographically distinct orientations that 
afford strong in-plane anisotropy of growth rates, leads to unusual interlaced spirals of the molecular layers and step bunches that advance at a rate limited by a crystallographically unique step edge in each layer. AFM measurements in the vicinity of the core permitted determination of the growth rates along the six crystallographically unique directions in a single molecular layer, corroborating the assignment of the slowest advancing directions as $\mathrm{A}(+)$ and $\mathrm{A}(-)$. This assignment was corroborated by the trend in the calculated binding energies for $L$-cystine at these step edges, which suggested a lower energy for $\mathrm{A}(+)$ and $\mathrm{A}(-)$, in which the S-S bond vector is parallel to the step edge. Additional calculations of the stereospecific replacement energies supported CDME incorporation at the A(+) step edge, consistent with the birefringence observed for $L$-cystine crystals grown in the presence of CDME. These observations illustrate a remarkable fidelity of the stereospecific binding of CDME at a unique crystal site in each molecular layer, with a highly preferred orientation with respect to the favored step edge. Careful inspection of the dislocation also revealed increased step spacing and decreased step velocities near the core, consistent with the large elastic stresses stemming from the large Burgers vector normal to the (0001) face, which spans six molecular layers. The observed critical lengths and step spacings were accounted for by the time required for a step segment to traverse the perimeter of a strained region around the center of the core, after which a new segment is created. Crystal growth inhibition by L-cystine dimethylester (CDME) is evident from step velocities that decrease with increasing CDME concentration and a widening of the dead zone, as well as a corresponding increase in step roughness. These observations are consistent with Cabrera-Vermilyea step pinning in which adsorbed inhibitor molecules block step propagation, thereby increasing the curvature of the step as it advances between pinning sites and decreasing the effective supersaturation of the solute. Collectively, these observations provide a more comprehensive understanding of the crystal growth and inhibition mechanisms associated with an unusual dislocation spiral morphology that reflects the coincidence of a crystallographic screw axis and a screw dislocation. Such investigations advance the understanding of phenomena that are critical to the design of therapeutic inhibitors at the molecular level as well as the formation of mixed crystals comprising intentionally incorporated guests into host crystals.

\section{ACKNOWLEDGEMENTS}

This work was supported by the MRSEC Program of the National Science Foundation under Award Number DMR-0820341. BK and MDW also acknowledge support from the NSF (CHE-0845526, DMR1105000, DMR-0906576) and New York University. ALR thanks the Australian Research Council for 
funding (DP140101776). ZZ is grateful to New York University for a Horizon Fellowship. The work also was supported by iVEC through the use of advanced computing resources available at iVEC@UWA.

\section{APPENDIX}

Derivation of equation (2) using the method from ref. 33. Let us consider one molecule tall (with height of $c / 6$ ) hexagonal island on the flat $L$-cystine (0001) surface. Assume two types of edges, $i=\mathrm{A}, \mathrm{B}$, having different lengths, $L_{i}$, and edge energies, $\gamma_{i}$ (Figure A1). The island area $\Omega=L_{\mathrm{A}} L_{\mathrm{B}} \sqrt{3}+L_{\mathrm{B}}^{2} \frac{\sqrt{3}}{2}$. The energy of the island is determined by the sum of bulk, $U_{V}$, and edge, $U_{\Omega}$, energies.

The bulk contribution $U_{V}=\frac{\Omega c Z}{6 \omega} \Delta \mu_{0}$, where $\omega=a^{2} c N_{A} \sin 60^{\circ}$ is the molar volume, $N_{A}$ is Avogadro's number, $a$ and $c$ are lattice constants of $L$-cystine, $Z=6$ is number of $L$-cystine molecules in the unit cell, and $\Delta \mu_{0}$ is the difference in chemical potentials (negative in case of growth).

The edge energy contribution $U_{\Omega}=4 L_{\mathrm{B}} \gamma_{\mathrm{B}}+2 L_{\mathrm{A}} \gamma_{\mathrm{A}}$. Applying standard energy minimization procedure with respect to the edge lengths $\frac{d\left(U_{V}+U_{\Omega}\right)}{d L_{i}}=0$ one can get the critical lengths of the hexagon edges (equation (2)):

$L_{c \mathrm{~A}}=\frac{\left(2 \gamma_{\mathrm{B}}-\gamma_{\mathrm{A}}\right) N_{A} a^{2}}{\Delta \mu_{0}}$ and $L_{c \mathrm{~B}}=\frac{\gamma_{\mathrm{A}} N_{A} a^{2}}{\Delta \mu_{0}}$. 


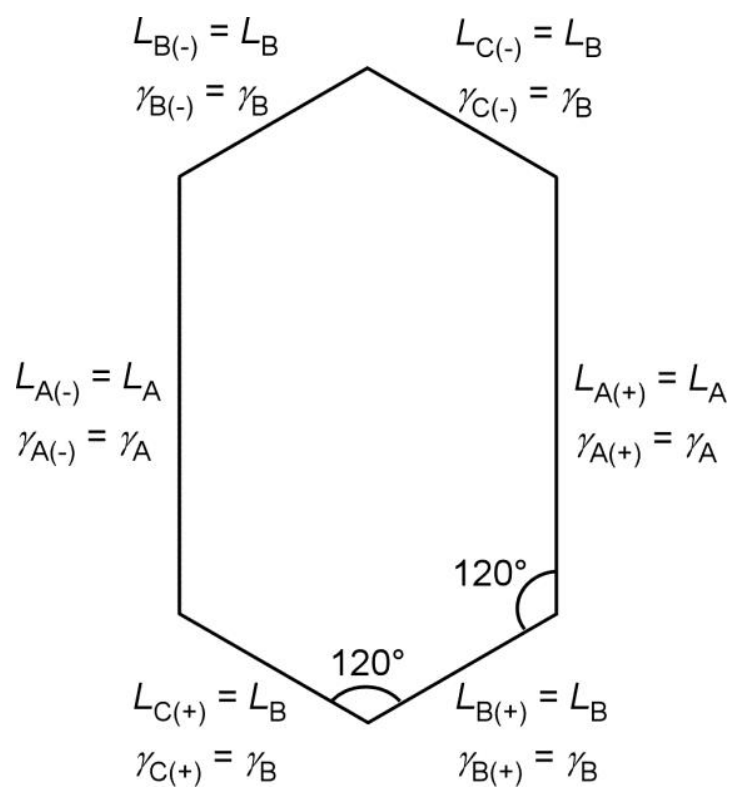

Figure A1. Assignment of side lengths and edge energies for hexagonal island on L-cystine (0001) surface.

\section{REFERENCES}

(1) Becker, G. Nephrology 2007, 12, S4-S10.

(2) Nakagawa, Y.; Asplin, J. R.; Goldfarb, D. S.; Parks, J. H.; Coe, F. L. J. Urol. 164, 1481-1485.

(3) Dolin, D. J.; Asplin, J. R.; Flagel, L.; Grasso, M.; Goldfarb, D. S. J. Endourol. 2005, 19, 429-432.

(4) Rimer, J. D.; An, Z.; Zhu, Z.; Lee, M. H.; Goldfarb, D. S.; Wesson, J. A.; Ward, M. D. Science 2010, 330, $337-341$.

(5) Berkovitch-Yellin, Z.; Van Mil, J.; Addadi, L.; Idelson, M.; Lahav, M.; Leiserowitz, L. J. Am. Chem. Soc. 1985, 107, 3111-3122.

(6) Weissbuch, I.; Addadi, L.; Leiserowitz, L. Science 1991, 253, 637-45.

(7) Jones, F.; Ogden, M. I. CrystEngComm 2010, 12, 1016-1023.

(8) De Yoreo, J. J.; Vekilov, P. G. In Biomineralization; Dove, P. M., De Yoreo, J. J., Weiner, S., Eds.; Mineralogical Society of America Geochemical Society: Washington, D.C., 2003; Vol. 54, p 57-93.

(9) Rashkovich, L. N.; De Yoreo, J. J.; Orme, C. A.; Chernov, A. A. Crystallogr. Rep. 2006, 51, 1063-1074.

(10) Rashkovich, L. N.; Petrova, E. V.; Shustin, O. A.; Chernevich, T. G. Phys. Solid State 2003, 45, 400-407.

(11) Fujiki, Y.; Tokunaga, N.; Shinkai, S.; Sada, K. Angew. Chem. Int. Ed. 2006, 45, 4764-4767.

(12) Carta, R.; Tola, G. J. Chem. Eng. Data 1996, 41, 414-417.

(13) Kallistratos, G.; Malorny, G. Arzneimittel-Forsch. 1972, 22, 1434-1444.

(14) Königsberger, E.; Wang, Z.; Königsberger, L.-C. Monat. Chem. 2000, 131, 39-45.

(15) Glazer, A. M.; Lewis, J. G.; Kaminsky, W. Proc. R. Soc. London A 1996, 452, 2751-2765.

(16) Kaminsky, W.; Claborn, K.; Kahr, B. Chem. Soc. Rev. 2004, 33, 514-525.

(17) Sun, H. Phys. Chem. B 1998, 102, 7338-7364.

(18) Gale, J. D.; Rohl, A. L. Mol. Simulat. 2003, 29, 291-341. 
(19) Gale, J. D.; Rohl, A. L. Mol. Simulat. 2007, 33, 1237-1246.

(20) Wang, J.; Wolf, R. M.; Caldwell, J. W.; Kollman, P. A.; Case, D. A. J. Comp. Chem. 2004, 25, 1157-1174.

(21) Vanquelef, E.; Simon, S.; Marquant, G.; Garcia, E.; Klimerak, G.; Delepine, J. C.; Cieplak, P.; Dupradeau, F.-Y. Nucleic Acids Res. 2011, 39, W511-W517.

(22) Oughton, B. M.; Harrison, P. M. Acta Crystallogr. 1959, 12, 396-404.

(23) Stoica, C.; van Enckevort, W. J. P.; Meekes, H.; Vlieg, E. J. Cryst. Growth 2007, 299, 322-329.

(24) van Enckevort, W. J. P.; Bennema, P. Acta Crystallogr. A 2004, 60, 532-541.

(25) Levitt, L. S. J. Phys. 1975, 49, 696-697.

(26) Hartman, P.; Perdok, W. G. Acta Crystallogr. 1955, 8, 525-529.

(27) Hartman, P.; Perdok, W. G. Acta Crystallogr. 1955, 8, 49-52.

(28) Hartman, P.; Perdok, W. G. Acta Crystallogr. 1955, 8, 521-524.

(29) Shtukenberg, A. G.; Zhu, Z.; An, Z.; Bhandari, M.; Song, P.; Kahr, B.; Ward, M. D. Proc. Nat. Acad. Sci. 2013, 110, 17195-17198.

(30) Teng, H. H.; Dove, P. M.; Orme, C. A.; De Yoreo, J. J. Science 1998, 282, 724-727.

(31) Hartman, P. J. Cryst. Growth 1980, 49, 157-165.

(32) Nielsen, A. E.; Söhnel, O. J. Cryst. Growth 1971, 11, 233-242.

(33) Lovette, M. A.; Browning, A. R.; Griffin, D. W.; Sizemore, J. P.; Snyder, R. C.; Doherty, M. F. Ind. Eng. Chem. Res. 2008, 47, 9812-9833.

(34) Sizemore, J.P.; Doherty, M.F. Cryst. Growth Des. 2009, 9, 2637-2645.

(35) Sizemore, J. P.; Doherty, M. F. J. Cryst. Growth 2010, 312, 785-792.

(36) van der Hoek, B.; van der Eerden, J. P.; Bennema, P. J. Cryst. Growth 1982, 56, 621-632.

(37) Chuong, C. J.; Zhong, P.; Preminger, G. M. J. Endourology 1993, 7, 437-444.

(38) Chickos, J.S.; Nichols, G. J. Chem. Inf. Comput. Sci. 2002, 42, 368-374.

(39) Acree, W.E. Thermochimica Acta 1991, 189, 37-56.

(40) Foreman, A. J. E. Acta Met. 1955, 3, 322-330.

(41) De Yoreo, J. J.; Land, T. A.; Rashkovich, L. N.; Onischenko, T. A.; Lee, J. D.; Monovskii, O. V.; Zaitseva, N. P. J. Cryst. Growth 1997, 182, 442-460.

(42) Derksen, A. J.; Enckevort, W. J. P. v.; Couto, M. S. J. Phys. D 1994, 27, 2580-2591.

(43) Celik, Y.; Graham, L. A.; Mok, Y.-F.; Bar, M.; Davies, P. L.; Braslavsky, I. Proc. Nat. Acad. Sci. 2010, $107,5423-5428$.

(44) Rashkovich, L. N.; Kronsky, N. V. J. Cryst. Growth 1997, 182, 434-441.

(45) Ristic, R. I.; DeYoreo, J. J.; Chew, C. M. Cryst. Growth Des. 2008, 8, 1119-1122.

(46) Chernov, A. A.; Rashkovich, L. N. J. Cryst. Growth 1987, 84, 389-393.

(47) Cabrera, N.; Vermilyea, D.; Doremus, R.; Roberts, B.; Turnbull, D. In Growth and Perfection of Crystals, Proceedings of the International Conference, Cooperstown, NY, August 1958; Wiley, New York 1958, pp. 393-410.

(48) Black, S. N.; Davey, R. J.; Halcrow, M. J. Cryst. Growth 1986, 79, 765-774.

(49) Thomas, T. N.; Land, T. A.; DeYoreo, J. J.; Casey, W. H. Langmuir 2004, 20, 7643-7652.

(50) Weaver, M. L.; Qiu, S. R.; Hoyer, J. R.; Casey, W. H.; Nancollas, G. H.; De Yoreo, J. J. ChemPhysChem 2006, 7, 20812084.

(51) Weaver, M. L.; Qiu, S. R.; Hoyer, J. R.; Casey, W. H.; Nancollas, G. H.; De Yoreo, J. J. J. Cryst. Growth 2007, 306, 135145. 
(52) Sleutel, M.; Van Driessche, A. E. S. Cryst. Growth Des. 2013, 13, 688-695.

(53) Sangwal, K. J. Cryst. Growth 1999, 203, 197-212.

(54) Kubota, N.; Mullin, J. W. J. Cryst. Growth 1995, 152, 203-208.

(55) De Yoreo, J. J.; Zepeda-Ruiz, L. A.; Friddle, R. W.; Qiu, S. R.; Wasylenki, L. E.; Chernov, A. A.; Gilmer, G. H.; Dove, P. M. Cryst. Growth Des. 2009, 9, 5135-5144.

(56) Sangwal, K. In Additives and Crystallization Processes: From Fundamentals to Applications; John Wiley \& Sons, Ltd: Chichester, 2007, p. 116.

(57) Sleutel, M.; Sazaki, G.; Van Driessche, A. E. S. Cryst. Growth Des. 2012, 12, 2367-2374.

(58) Weaver, M. L.; Qiu, S. R.; Friddle, R. W.; Casey, W. H.; De Yoreo, J. J. Cryst. Growth Des.2010, 10, $2954-2959$.

(59) van Enckevort, W. J. P.; van den Berg, A. C. J. F. J. Cryst. Growth 1998, 183, 441-455.

(60) Kahr, B.; McBride, J. M. Angew. Chem. Int. Ed. 1992, 31, 1-26.

(61) Shtukenberg, A. G.; Punin, I. Yu. (Kahr, B. Ed.) Optically Anomalous Crystals; Springer: Dordrecht, 2007.

(62) Batsanov, S. S.; Batsanov, A. S. Introduction to Structural Chemistry; Springer: Dordrecht, 2012; p. 522. 


\section{For Table of Contents Use Only}

Dislocation-actuated Growth and Inhibition of Hexagonal $L$-cystine Crystallization at the Molecular Level

Alexander G. Shtukenberg, Laura N. Poloni, Zina Zhu, Zhihua An, Misha Bhandari, Pengcheng Song, Andrew L. Rohl, Bart Kahr, and Michael D. Ward

\section{Table of Contents Graphic}

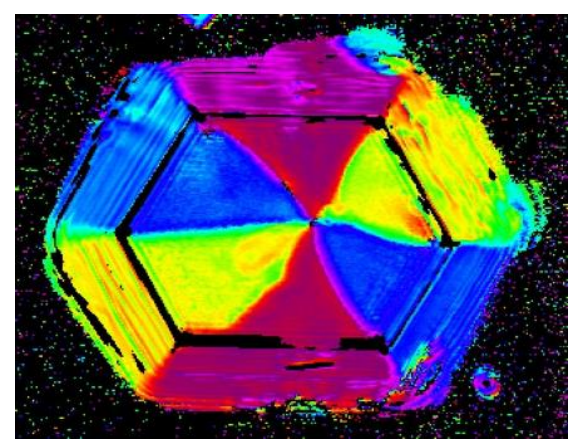

\section{Synopsis}

The crystallization kinetics of $L$-cystine and effect of tailored additive $L$-cystine dimethylester were studied using in situ atomic force microscopy. Anisotropy of growth kinetics and high magnitude oflarge Burgers vector lead toresult in a complex character of dislocation growth. $L$-cystine dimethylester inhibits growth in accordance with the Cabrera-Vermilyea model and gets incorporated into a crystal structure with a high selectivity. 